\title{
White matter changes in patients with mild traumatic brain injury: MRI perspective
}

\begin{abstract}
This review focuses on white matter (WM) changes in mild traumatic brain injury (mTBI) as assessed by multimodal MRI. All the peer reviewed publications on WM changes in mTBI from January 2011 through September 2016 are included in this review. This review is organized as follows: introduction to $\mathrm{mTBI}$, the basics of multimodal MRI techniques that are potentially useful for probing the WM integrity, summary and critical evaluation of the published literature on the application of multimodal MRI techniques to assess the changes of WM in $\mathrm{MTBI}$, and correlation of MRI measures with behavioral deficits. The MRI-pathology correlation studies based on preclinical models of $\mathrm{mTBI}$ are also reviewed. Finally, the author's perspective of future research directions is described.
\end{abstract}

First draft submitted: 10 December 2016; Accepted for publication: 10 February 2017; Published online: 22 March 2017

Keywords: MRI $\bullet \mathrm{MRI}-$ pathology correlation $\bullet \mathrm{MRS} \bullet \mathrm{mTBI} \bullet \mathrm{WM}$ injury

About $2 \%$ of the US population is affected by traumatic brain injury (TBI) which results in an annual healthcare cost of $\$ 76.5$ billion [1]. Based on clinical symptoms, TBI can be categorized as either mild or moderate or severe [2]. Focal TBI generally results in contusion or laceration to cortical and subcortical structures and intracranial bleeding and results in severe TBI. In contrast, diffuse injury is the result of stretching and tearing of axons due to linear and angular acceleration/deceleration. Linear acceleration causes less severe axonal shearing than angular acceleration/deceleration [3]. Traumatic axonal injury (TAI) is thought to be a major pathologic event in TBI and may be responsible for much of the observed cognitive deficits [4-12]. Diffuse axonal injury (DAI) may be considered as a subset of TAI. Diffuse axonal injury has some histopathological characteristics that occur immediately and within hours and days of injury. Axons in white matter (WM) are vulnerable to diffuse injury because of their small diam- eter $(\sim 3 \mu)$ and long projections. DAI could result in mechanical breaking of the axonal cytoskeleton that could affect axonal transportation which in turn affects communication between different brain structures. WM pathology, and especially that detected with neuroimaging, is also influenced by other factors such as blood flow/edema, metabolic aberrations, excitotoxicity and secondary degeneration, Wallerian degeneration, and other factors, which occur under the umbrella of TAI and coalesce over time, not just what occurs within the acute time frame. Depending on the severity of DAI, TBI victims could experience cognitive and behavioral deficits. Advances in biomechanical modeling identified WM pathways that are most vulnerable to stretch and shear injury. These pathways include corona radiata (CR), internal capsule (IC), cerebral peduncle (CP) and corpus callosum (CC) [13-15].

About $80 \%$ of TBI incidents in military and civilian populations are classified as mild (mTBI) [16], which is considered to be a major
Ponnada A Narayana

Department of Diagnostic \& Interventional Imaging, McGovern Medical School, University of Texas Health Science Center at Houston, Houston, TX 77030, USA Tel.: +1 7135007677

ponnada.a.narayana@uth.tmc.edu
Future
Medicine $\mathrm{fS}$ 
public health problem [17-19]. mTBI could result from any number of mechanisms that include blunt trauma resulting from falls and vehicular accidents, collisions such as those experienced in sports related activities, and blast, experienced mainly by army personnel. The diagnostic criteria for mTBI typically include loss of consciousness (LOC) for less than $30 \mathrm{~min}$, amnesia for a period of less than $24 \mathrm{~h}$, and a score of $13-15$ on the Glasgow Coma Scale [20]. Majority of the post-mTBI symptoms are resolved spontaneously within days to a few weeks. In about $30 \%$ of mTBI patients postconcussion symptoms (PCS) persist over a period of several months or longer [21]. TRACK-TBI study indicates that about $22.4 \%$ of the mTBI subjects are not completely functional at 1 year post injury [22]. Notwithstanding the word 'mild,' mTBI patients experience somatic, affective, and cognitive symptoms, collectively referred to as PCS or postconcussional disorder that compromises the quality of life. Understanding the reasons for the prolonged PCS in these patients has important implications in the management of mTBI.

Concussion has recently attracted considerable attention because of the injuries suffered by high school, college and professional players [23,24], and military personnel [25]. Concussion is generally considered to be the mildest form of mTBI. Concussion and mTBI have similar and overlapping symptoms [26]. As pointed out elsewhere all concussions are mild TBIs, though all mTBIs are not concussions' [27]. Nevertheless, these two terms are used interchangeably in the published literature. In this review, consistent with most published literature, the terms concussion and mTBI are used interchangeably. In this review, whenever possible, we tried to follow the recommended definition of the postinjury phase: acute $<1$ day; subacute: 1 day-1 week; postacute 1 week- 6 months; chronic: $>6$ months [28].

The commonly used techniques for evaluating WM injury in mTBI include immunohistochemistry, electron microscopy and neuroimaging. The first two techniques require tissue samples and are not practical for routine use in clinical studies. The commonly used noninvasive neuroimaging techniques for evaluating injury in mTBI include computerized tomography (CT) and MRI. Among these two, MRI is most commonly used for evaluating mTBI because of its superior soft tissue contrast and its multimodal nature that can provide anatomical, structural, functional, physiological and metabolic information [29-32]. MRI also has the ability to detect subtle pathology in mTBI even when the clinical symptoms appear to return to normal values [30,33-36]. Conventional MRI based on T1-, T2-weighted and FLAIR images is of limited use in detecting WM injury in mTBI $[29,37,38]$. As discussed below, published literature also indicates the potential of more recent MRI-based techniques that include, diffusion tensor imaging (DTI), magnetization transfer ratio (MTR), susceptibility weighted imaging (SWI), myelin water imaging, ultrashort echo time, and proton magnetic resonance spectroscopy (MRS) in detecting WM injury in mTBI. Among these, DTI has shown considerable promise in identifying underlying neuropathology in mTBI [29]. This review does not cover functional MRI (fMRI) [39] or positron emission tomography (PET) [40] since the focus of these modalities is mainly on gray matter.

The role of advanced neuroimaging techniques in TBI were recently reviewed [41]. The TRACK-TBI study also demonstrated the superiority of advanced MRI in predicting outcome in mTBI at 3 and 6 months postinjury time [42]. The published literature on WM injury in human brain using different MRI-based techniques is summarized in Table 1. Since prior reviews summarized published literature till 2011, we included only publications listed in Pubmed from January 2011 through September 2016. Inclusion of publications in 2011 provides an overlap with the previous reviews. We also summarize in Table 2 the review articles on mTBI published from January 2011 to September 2016 with a strong neuroimaging component. The basics of these techniques are briefly reviewed, followed by their application to WM injury in mTBI.

The pathophysiology of mTBI at the cellular level can be found in a number of excellent reviews [3,97-99] and will not be included here. Since the main focus of this review is on WM, the effect of mTBI on cortical and subcortical structures is not included in this review. Also the effect of repetitive concussions will not be explicitly discussed. Consistent with this journal's recommendation, only references to the works published in the last 5 years or so are included. Thus many important original publications that appeared earlier than 5 years are not referenced. There are a number of excellent reviews that covered earlier studies and references to these review articles are provided at the appropriate place. All relevant publications between January 2011 and September 2016 were identified by searching PubMed using various queries that are combinations of the key words, 'mild traumatic brain injury', 'traumatic brain injury', 'concussion', 'sports concussion', 'MRI', 'MRS', 'DTI', 'white matter hyperintensities and pathology'. Both human and preclinical studies were included in this search.

\section{MRI techniques relevant to investigate WM injury in $\mathrm{mTBI}$}

A number of MRI-based modalities have been used to probe the WM injury in mTBI and to determine pos- 
sible association between the MRI measures and cognitive, behavioral, and neuropsychiatric disorders in mTBI $[38,62,95,100]$. Such an association could be helpful in the management of mTBI victims.

\section{Diffusion tensor imaging}

DTI has become the preferred modality for detecting and characterizing WM injury in mTBI. The WM consists of axonal bundles and water preferentially diffuses along the length of axons because of the barriers such as myelin, in other words, diffusion is anisotropic. DTI exploits the directional dependence of water diffusion in tissue to probe tissue microstructure. In contrast, water diffusion in GM is nearly isotropic. Anisotropic diffusion is described by a $3 \times 3$ symmetric diffusion matrix. All six independent elements of the diffusion matrix need to be determined for complete characterization of diffusion anisotropy. The diffusion matrix elements are determined by acquiring diffusion-weighted images (DWIs) in the presence magnetic field gradients. At least six DWIs are required, with noncollinear gradient directions. In practice more gradient directions are needed for robust determination of the diffusion matrix. In addition, MRI data have to be acquired without the application a diffusion gradient (commonly referred to as b0 images). Because DWI is sensitive to motion, it is important to ensure that DWIs are not corrupted by other motions, such as respiration. Therefore, DWIs are acquired using rapid sequences such as echo planar imaging or spiral sequences. Three principal diffusivities, $\lambda_{1}, \lambda_{2}$ and $\lambda_{3}$ (also known as eigenvalues) and three eigenvectors are estimated from the DWIs. These are needed for generating the fiber tracts. By convention, $\lambda_{1}$ has the largest value and is referred to as the longitudinal or axial diffusivity (AD). The average of $\lambda_{2}$ and $\lambda_{3}$ is referred to as the radial or transverse diffusivity (RD). The average of the three eigenvalues is referred to as the mean diffusivity (MD). The fractional anisotropy (FA), a dimensionless quantity, is the most commonly used measure of diffusion anisotropy and is derived from the three eigenvalues. FA varies from 0 to 1 , with 1 representing perfect anisotropy and 0 representing complete isotropy. Depending on the WM tract, the value of FA is typically between 0.4 and 0.8 with the FA value of cerebrospinal fluid (CSF) close to 0.0 . The GM which is mainly made of cell bodies has an FA value of $<0.2$. A lower $F A$ value in response to injury is thought to represent compromised WM. However, this is not always true in the presence of crossing fibers within the imaging voxel. For example, if one of the crossing fibers is compromised while others are intact, an increased FA is expected [101]. Thus caution should be exercised in interpreting altered FA values. FA can be affected both by the axonal and myelin state and is therefore less specific about the pathologic underpinnings of the injury. It is generally thought that $\mathrm{AD}$ and $\mathrm{RD}$ reflect axonal and myelin integrity, respectively, and therefore may have better pathologic specificity than FA. However, recent DTI-histology correlative studies do not completely support this contention [102].

Another measure that can be derived from the DWIs is Shannon entropy (SE). SE is based on the information theory and is a measure of diffusion anisotropy. Unlike DTI, SE is derived from DWIs without assuming any diffusion model. SE is high for anisotropic structures such as WM and low for isotropic structures such as CSF. SE can reveal tissue anisotropy that may not be apparent on FA. SE appears to be more sensitive to axonal density and less affected by fiber orientation than FA. Based on correlation with histology, SE appears to detect axonal remodeling that occurs in response to neurological injury [103].

\section{DTI acquisition \& analysis}

The acquisition parameters used for DTI can affect the most commonly used scalar variables, FA and MD [104-108]. The acquisition parameters include the number of gradient directions and the gradient scheme, the diffusion sensitization (commonly denoted by b), signal-to-noise-ratio (SNR), spatial resolution, field strength and even the scanner hardware and software. The number of gradient directions and spatial resolution affect FA, while diffusion sensitization (b) affects MD [108]. This dependence on the acquisition parameters makes it difficult to compare the diffusion results across different studies. Based on published studies, the reproducibility and repeatability of FA and MD improve with the number of gradient directions [106]. There is a general consensus that 30 gradient directions with a b value of approximately $1000 \mathrm{~s} \mathrm{~mm}^{-2}$ provides robust results.

The commonly used DTI analysis techniques can be classified into: whole brain histogram, region-ofinterest (ROI), voxel-based and tractography. Each one of these methods has its own advantages and disadvantages. The whole brain histogram analysis is quite robust and simple. However, it does not provide any spatial information. The ROI analysis allows testing hypothesis that is specific to tract and is also useful for following individual subjects longitudinally. However, it requires a priori knowledge about region that needs to be examined and may introduce bias. Voxel-based analysis does not require a priori knowledge and is independent of any hypothesis. It provides information over the whole brain while retaining the spatial information. However, it could introduce errors due to nonlinear image registration and is also prone to 


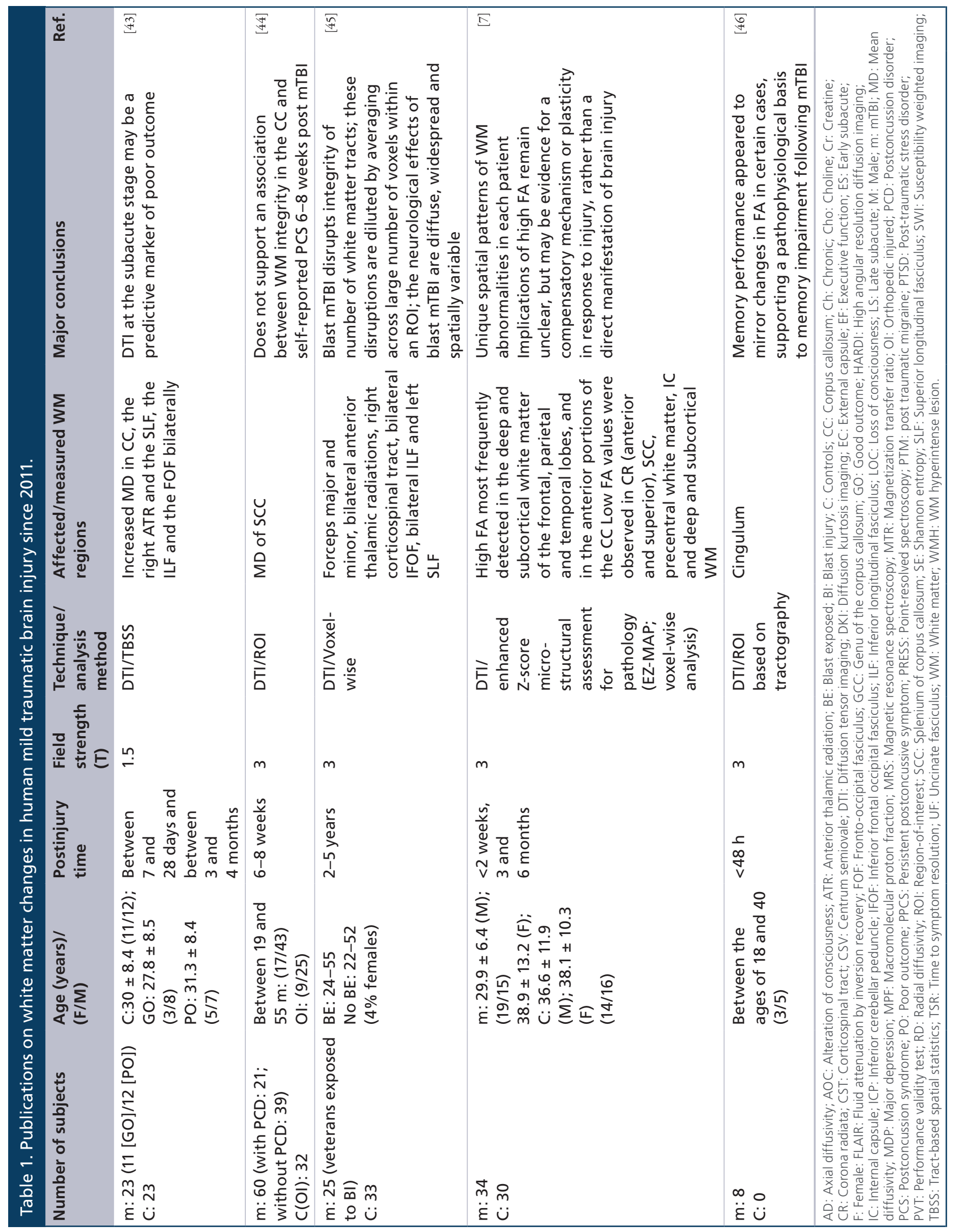




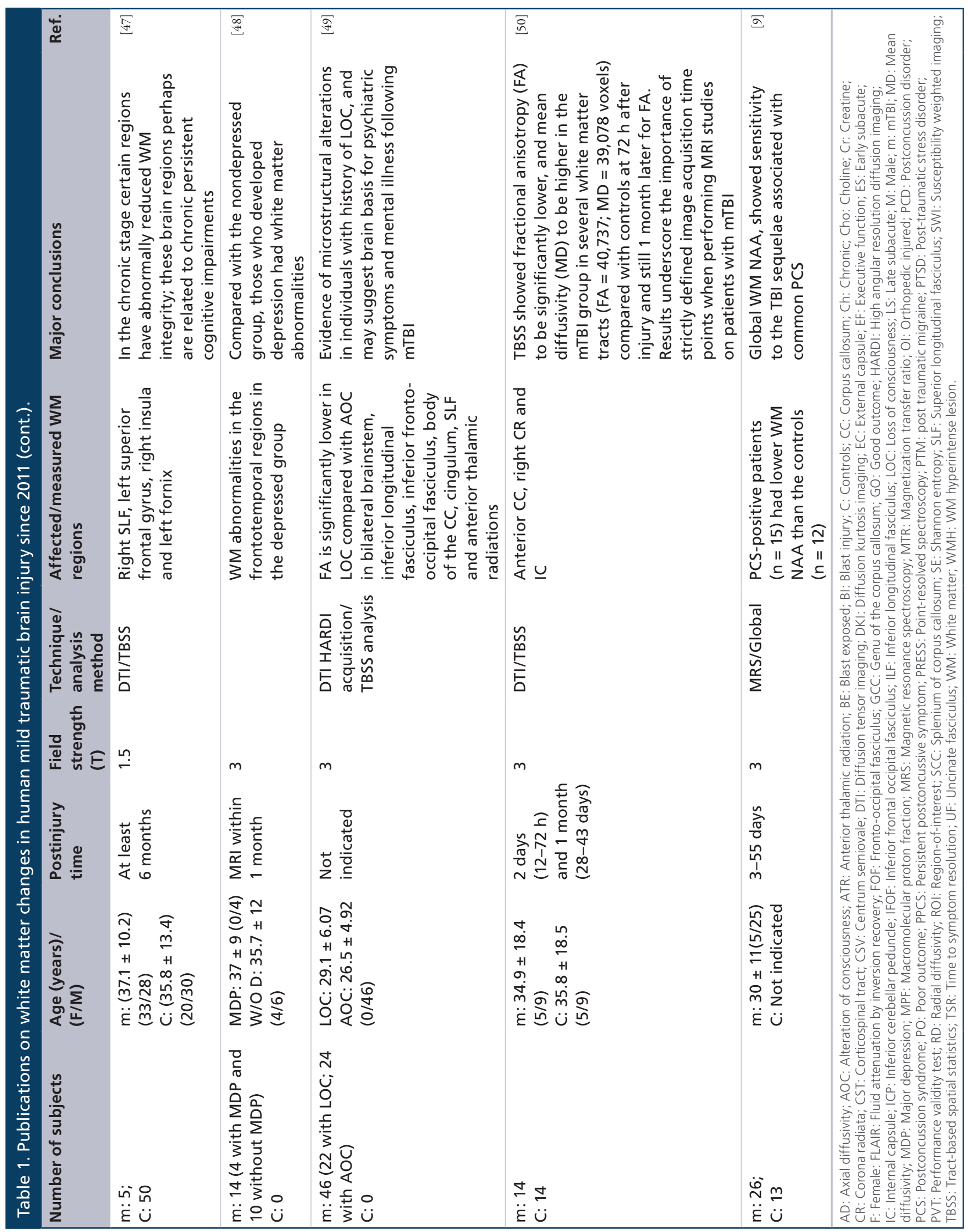




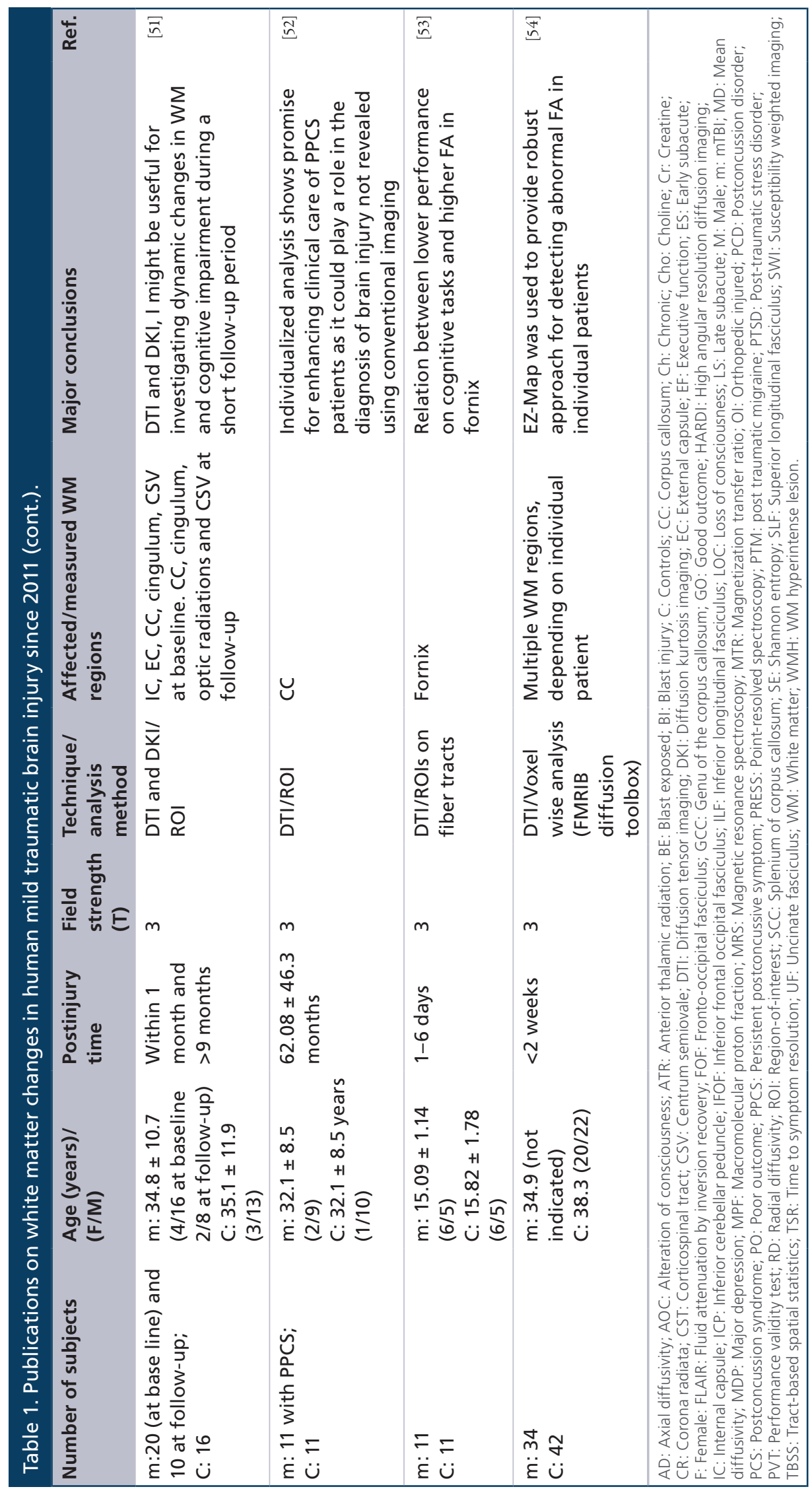




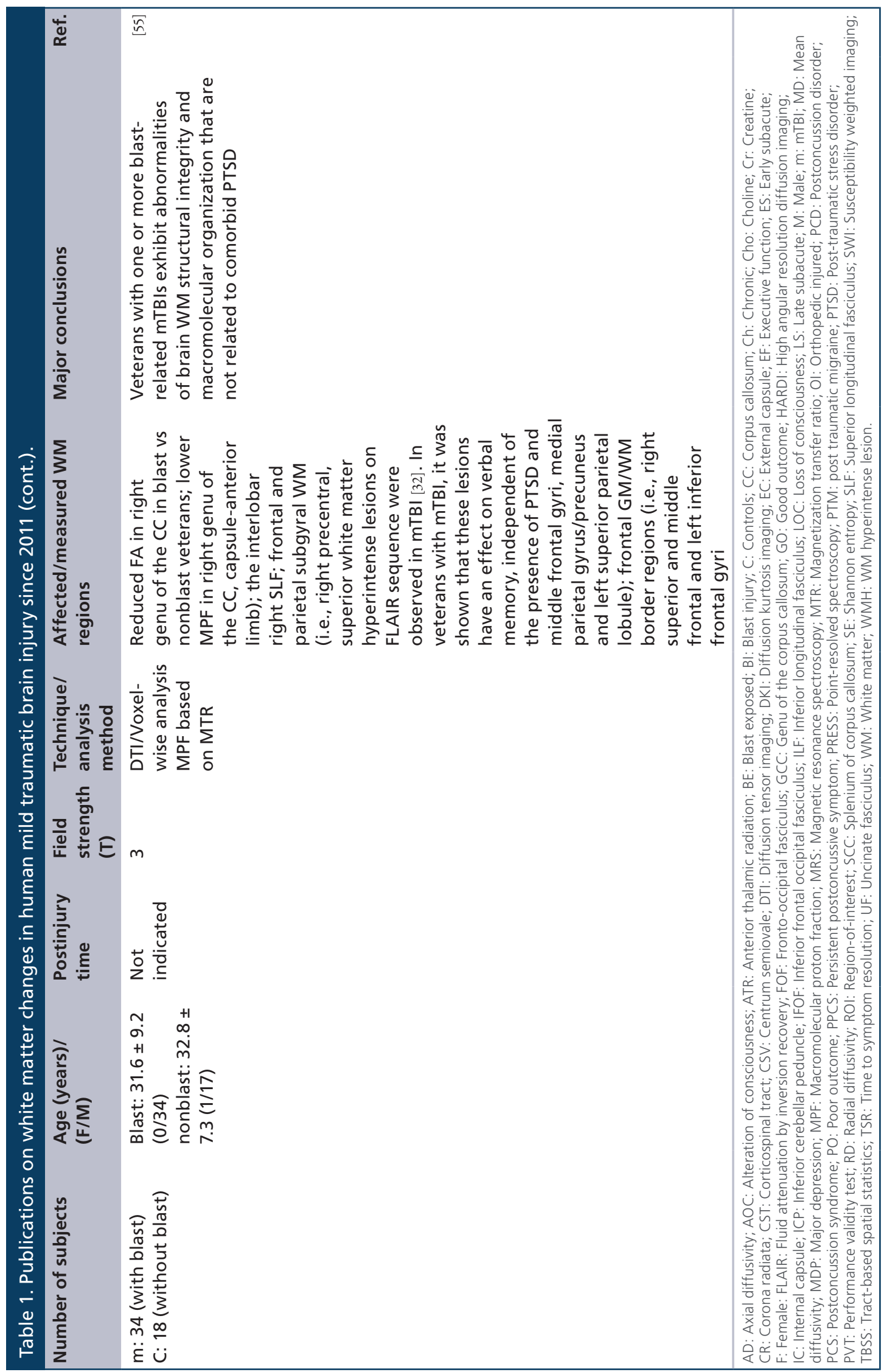




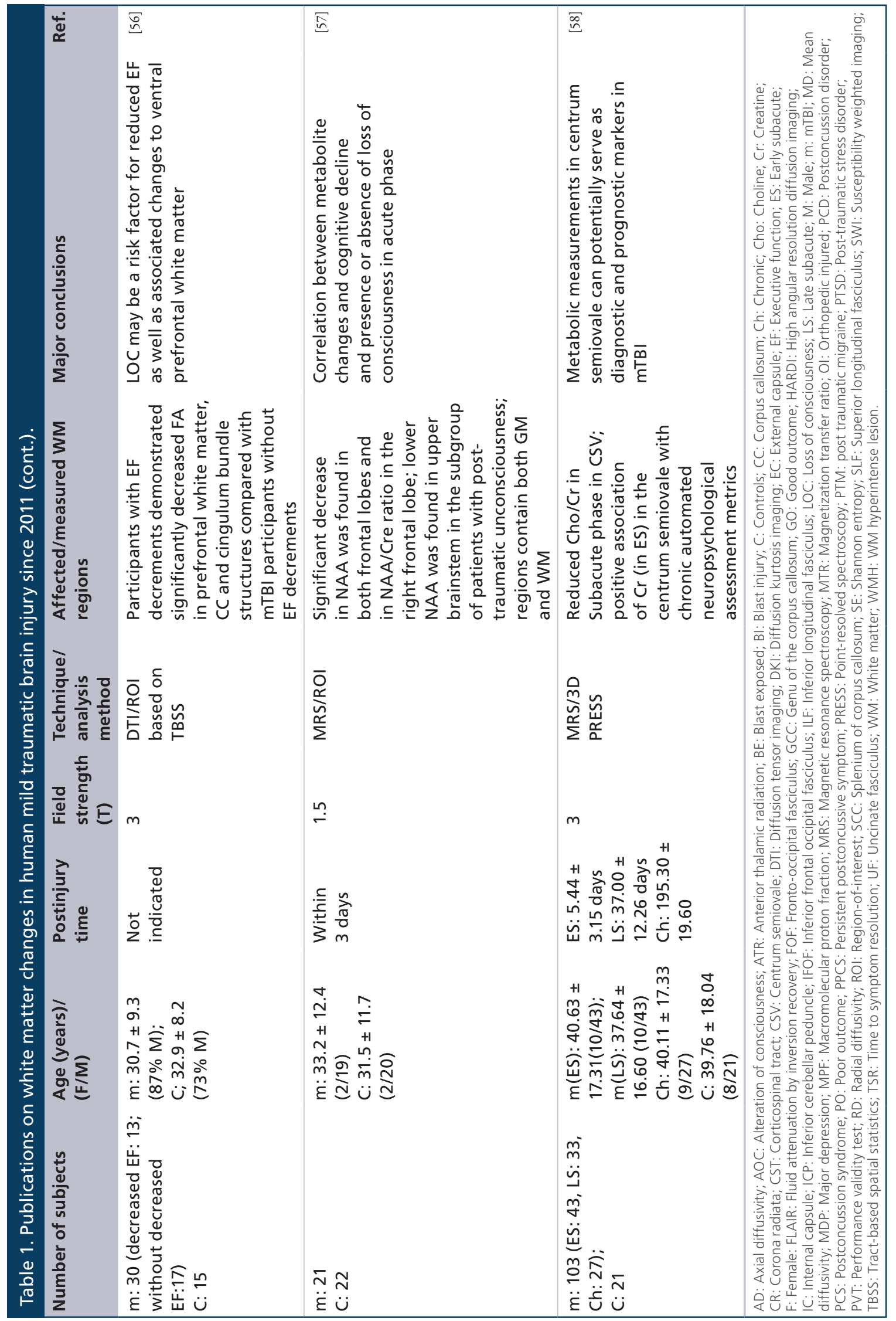




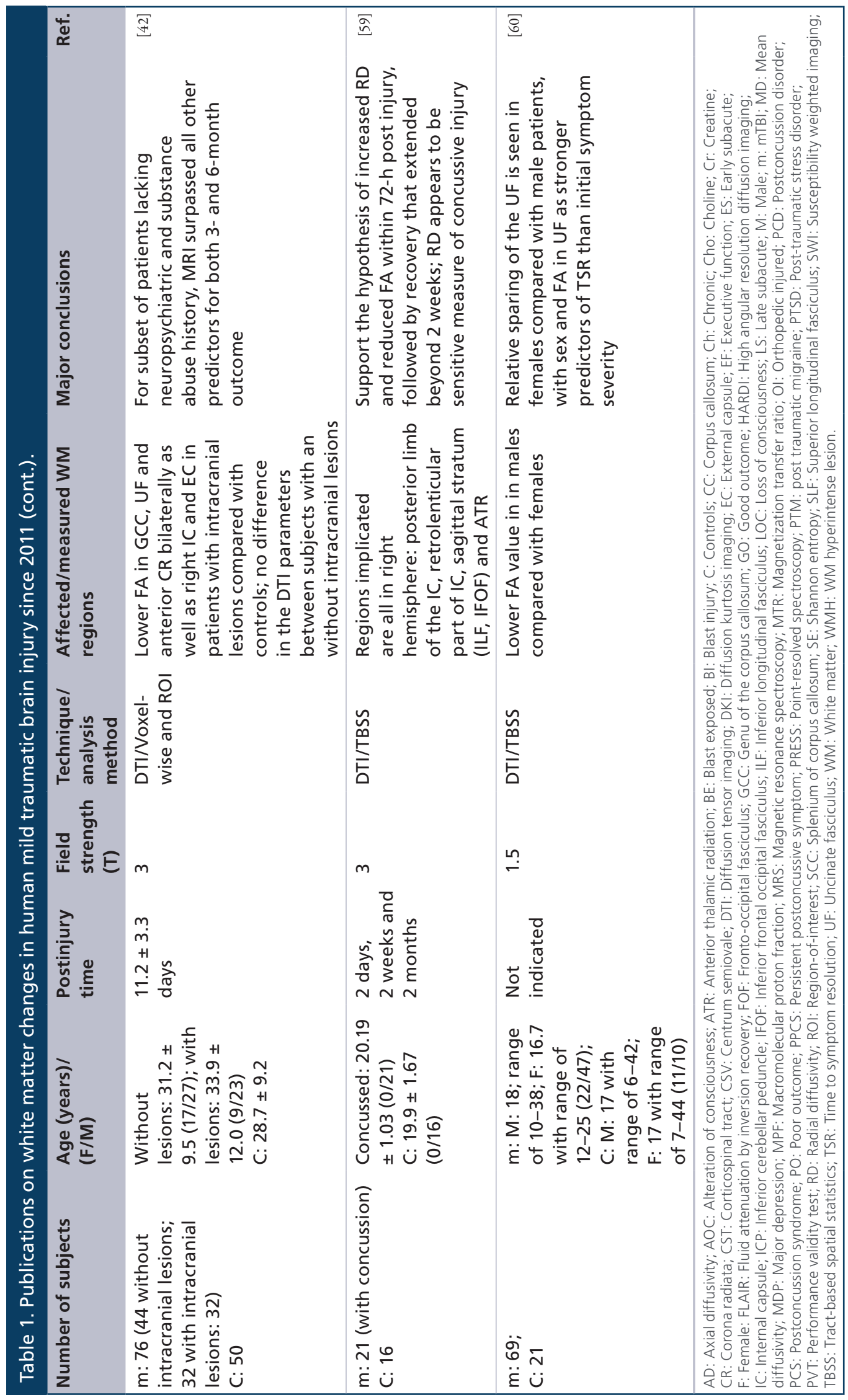




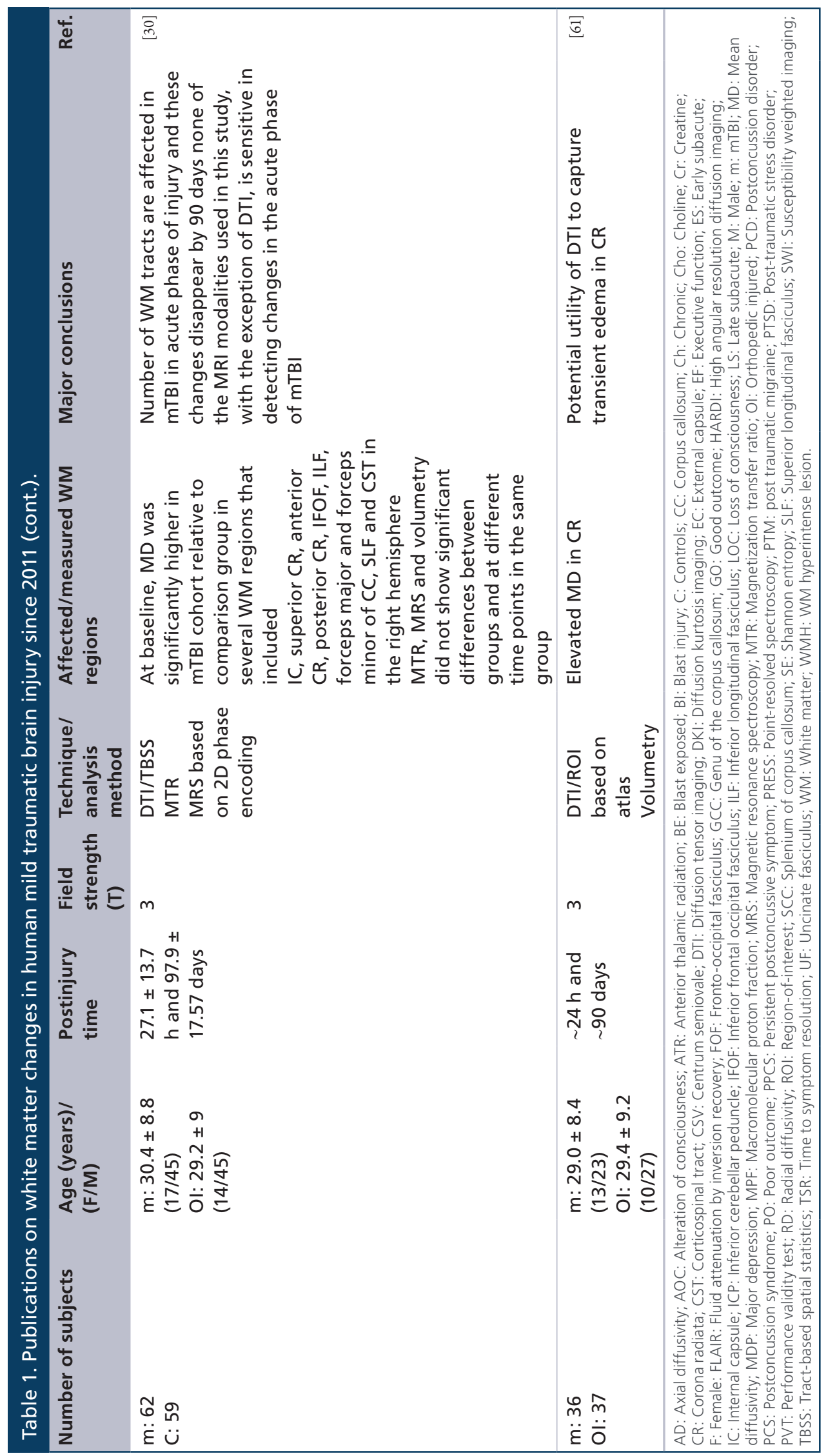




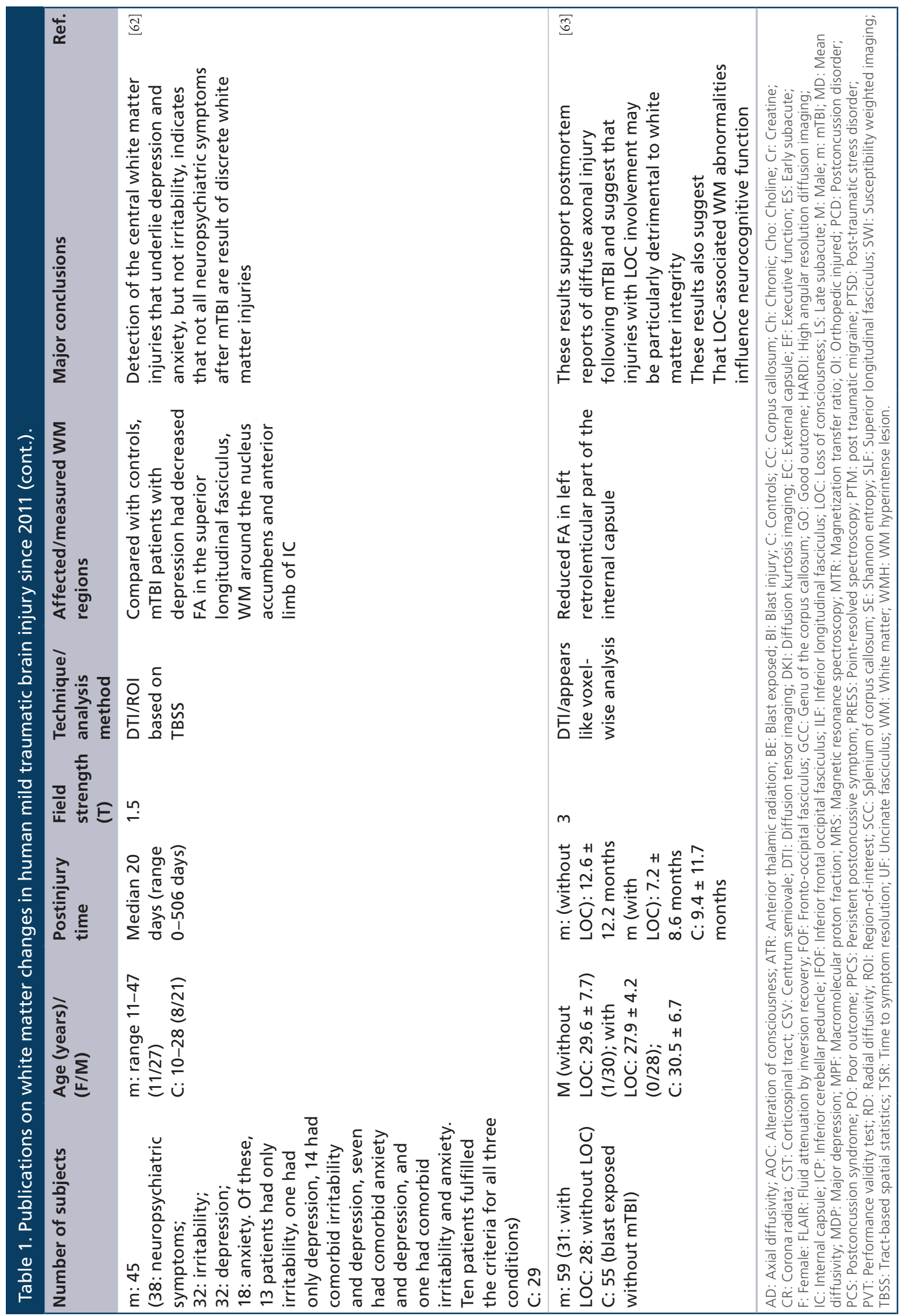




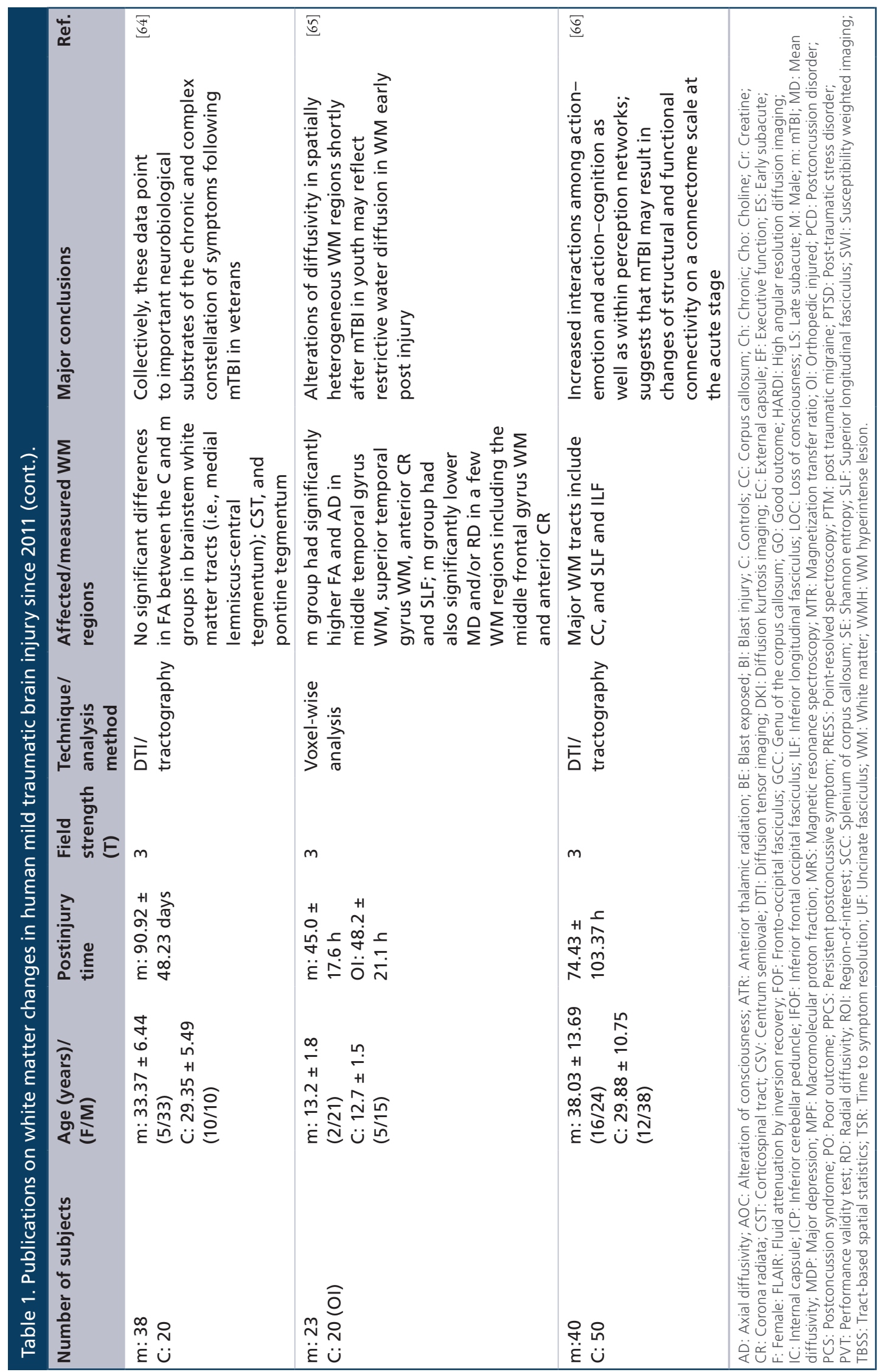




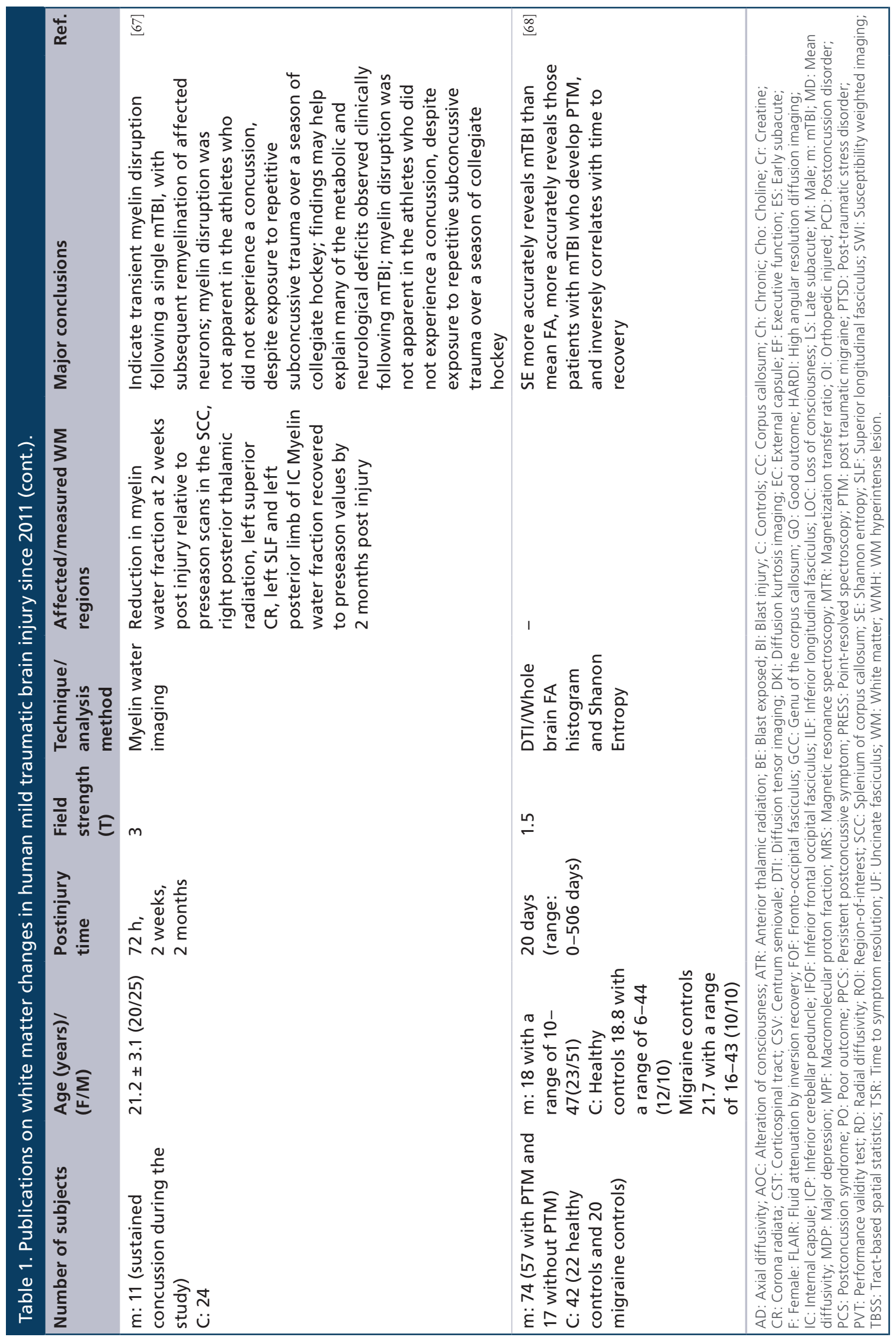




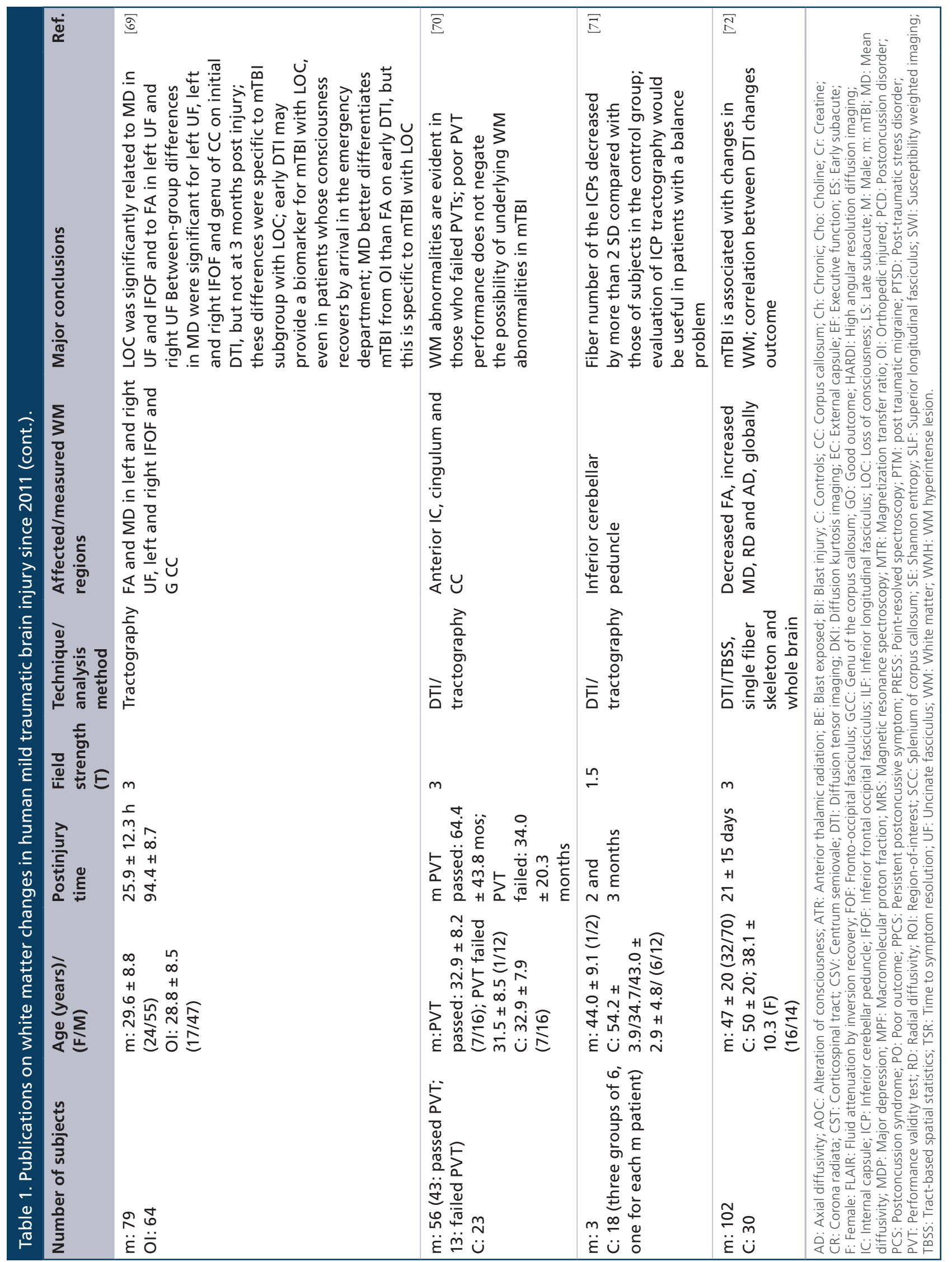




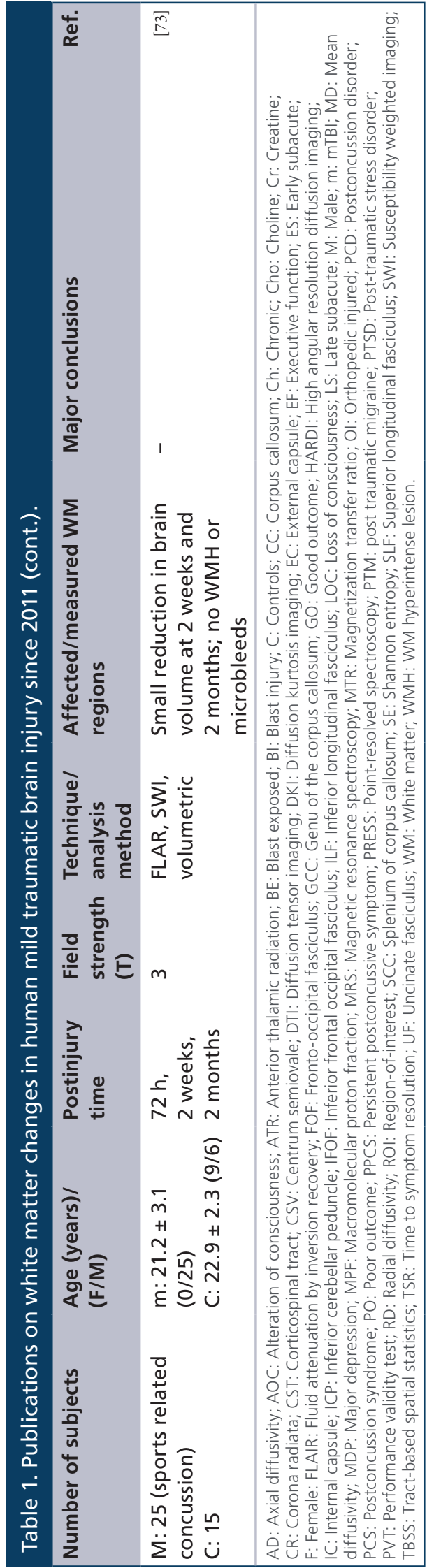

local noise in the images. This method also requires image smoothing to reduce image misalignment errors from image registration. It is not easy to objectively determine the extent of required smoothing. TBSS (tract-based spatial statistics) is a popular technique that is representative of voxel-based analysis. It is most suitable for group analysis. In order to minimize registration errors, the fiber tracts are skeletonized. TBSS includes only major WM tracts and the information about the tract edges is lost. However, TBSS analysis includes stricter threshold to account for multiple comparisons and may have less statistical power than the ROI analysis. Tractography based analysis has become popular with the availability of multiple software packages. The diffusion measures can be determined for the whole tract or regionally along the length of the tract. The results of this analysis are dependent on the quality of tractography.

The SNR, voxel resolution and the complexity of the fiber arrangements and the tract curvature can significantly affect the quality of tractography [109]. Tractography results appear to be affected both by the algorithms and the software packages used. For example, in one study it was shown that both deterministic and probabilistic tractography algorithms (the most widely used algorithm) result in 'systematically unreliable and clinically misleading information' [110]. However, both algorithms work fairly well for the core of the tracts. A recent study compared qualitatively and quantitatively the results obtained with four different software packages: Brainance (Advantis Medical Imaging, Eindhoven, The Netherlands), Philips FiberTrak (Philips, Best, The Netherlands), DSI Studio [111], NordicICE (Nordic NeuroLab, Bergen, Norway) [112]. This study showed both qualitative and quantitative agreement for some tracts such as forceps major and forceps minor, but poor agreement for cingulum bundle, superior longitudinal fasciculus, inferior fronto-occipital fasciculus, corticospial tracts and superior longitudinal fasciculus. All these studies clearly point out the need for critical evaluation of the tractography based methods, especially at tract locations close to the cortex.

Finally the choice of the method for the analysis of the DTI should be chosen based on the purpose of the study.

\section{Advanced diffusion imaging techniques}

Majority of the published literature is based on the assumption that diffusion follows Gaussian distribution and that the DTI data are analyzed using a single tensor model. However, diffusion deviates from 
Table 2. Reviews and meta-analyses of neuroimaging in mild traumatic brain injury.

Comments

Reviews the role of neuroimaging in sports-related concussion

Reviews the implications of DTI findings in mTBI

Focuses on DTI of frontal lobe

Focuses on the pathophysiologic role of stretch injury and relation to neuroimaging findings

Reviews the incidents of $\mathrm{mTBI}$ and objective radiological measures

Meta-analysis of voxel-based DTI

Reviews DTI application to sports-related concussion

Reviews DTI application to mTBI

Summarizes advanced neuroimaging findings in neuropsychological outcome in mTBI

Reviews neuroimaging studies to highlight the spectrum of acute to chronic time scales and provides meta-analyses to examine the role of MRI for studying both structure and function in $\mathrm{mTBI}$

Reviews the role of MRS in sports-related concussion

Summarizes evidence of neurodegeneration following TBI

Reviews white matter changes in sports related concussion

Reviews the application of advanced MRI in pediatric TBI

Reviews the application of high-resolution tractography to investigate white matter injury

Reviews the role of MRI in TBI

Reviews the role of, DTI, SWI, fMRI and MRS in MTBI

Reviews the results of advanced MRI in blast injury

Reviews the correlation between neuropathology and cognitive and behavioral deficits

Summarizes the long-term consequences of TBI

Reviews the application of MRI to MTBI

Summarizes the results of advanced MRI in MTBI

Reviews the role for neuroimaging in concussion

Meta-analysis of voxel-based (TBSS) in mTBI

Reviews the pitfalls of DTI in MTBI

Reviews the role of neuroimaging in youth sports

Provides a summary of clues to white matter injury repair

Reviews the MRI and CT findings in $\mathrm{MTBI}$, including sports-related concussion

Summarizes the application of multimodal neuroimaging in mTBI

DTI: Diffusion tensor imaging; fMRI: Functional MRI; mTBI: Mild traumatic brain injury; MRS: Magnetic resonance spectroscopy; PCS: Postconcussion syndrome; SWI: Susceptibility weighted imaging; TBSS: Tract-based spatial statistics.

Gaussian behavior in brain which has a complex tract arrangement. Diffusion kurtosis imaging (DKI) [113] to some extent accounts for the non-Gaussian behavior of water diffusion. DKI also helps calculate axonal water fraction and assessment of axonal and/or myelin loss [114]. Conventional DTI is typically acquired at $b$ $=0$ and approximately $1000 \mathrm{~s} \mathrm{~mm}^{-2}$, with multiple gradient orientations. In contrast, DKI data are acquired at multiple b-values with multiple gradient orientations. To reduce the acquisition times, it is common to acquire DKI data with two nonzero b-values. Typi- cally used b-values in DKI are approximtely $1000 \mathrm{~cm}^{2}$ $\mathrm{s}^{-1}$ and $\sim 2000 \mathrm{~cm}^{2} \mathrm{~s}^{-1}$. The commonly derived DKI measures are mean kurtosis and axial and radial kurtosis. The DKI and DTI measures are mathematically related [115].

Using conventional DTI, it is difficult to resolve crossing and kissing fibers. The inability to resolve crossing fibers makes the interpretation of diffusion measurements difficult. These problems can be overcome to some extent using advanced diffusion imaging. High angular resolution diffusion imaging (HARDI) is used 
to resolve crossing fibers (see, e.g., [116]). HARDI data are acquired using a larger number of gradient directions and orientations. HARDI is particularly useful for visualizing complex tract arrangement in brain. HARDI is typically acquired at multiple $b$ values and large number of gradient directions. The orientation direction function is determined from the HARDI data for determining the tract direction. The anisotropy is generally expressed as diffusion anisotropy and generalized FA [116].

It is worth pointing that from the acquisition point of view DTI is a subset of DKI and HARDI. The DTI measures can be derived both from the DKI and HARDI data. The longer scan times are limitations of HARDI and DKI.

\section{Susceptibility-weighted imaging}

Magnetic susceptibility, $\chi$, is a basic material property that is a measure of the ability of an applied magnetic field to magnetize the material. Mathematically it can be represented by the equation $\mathrm{M}=\chi \mathrm{B}$, where $M$ is the magnetization induced by the magnetic field B. Susceptibility is positive for paramagnetic and ferromagnetic materials and negative for diamagnetic substances, such as water. Susceptibility is a dimensionless quantity and different substances have different values. For example, the $\chi$ value of pure water is $-9.05 \times 10^{-6}$. In contrast, the $\chi$ values of deoxygenated and oxygenated red blood cells are $-6.52 \times 10^{-6}$ and $-9.19 \times 10^{-6}$, respectively. In comparison, ferritin loaded fully with 4500 ferric ions has a positive $\chi$ of $520 \times 10^{-6}$. Tissues and biological substances have different susceptibilities. SWI exploits the differences in the susceptibility values between different tissues. SWI is most commonly used for visualizing veins and hem- orrhage in the tissue. The principles and applications of SWI are recently reviewed [117]. It is one of the most sensitive techniques for visualizing microhemorrhage, thought to be an indicator of DAI. The sensitivity of SWI increases with the magnetic field strength.

\section{Magnetization transfer imaging}

Tissue contains at least two types of water pools: mobile and tightly bound to macromolecules such as myelin. The MR signal mainly arises from the mobile water protons. Because of the short T2 relaxation time, the tightly bound water proton signal is very broad and cannot be directly detected on MRI. However, the presence of tightly bound water protons can be detected indirectly by transferring magnetization from the bound water protons to the mobile water protons using an off-resonance radio frequency (RF) pulse whose frequency is slightly different $(\sim$ few $\mathrm{kHz})$ from that of the mobile water proton frequency. Because of the large line width of the magnetic resonance signal from the bound water protons, this off-resonance pulse can excite the bound water protons without directly affecting the mobile water proton signal. This transfer of magnetization from one pool to another is referred to as magnetization transfer imaging (MTI). In a typical MTI experiment the MR signal is acquired with $\left(\mathrm{M}_{\mathrm{on}}\right)$ and without $\left(\mathrm{M}_{\text {off }}\right)$ the off-resonance pulse. The MTR is calculated on a pixel-by-pixel basis as MTR = $\left(\mathrm{M}_{\text {off }}-\mathrm{M}_{\text {on }}\right) / \mathrm{M}_{\text {off }}$ The MTR signal depends on the concentration of the bound water molecules, among others. Since myelin is the major macromolecular entity in brain, MTR is an indirect measure of myelin concentration. The MTR signal also depends on the amplitude and frequency of the off-resonance pulse. MTR is a semiquantitative technique that is affected by the

Table 3. Frequency of white matter tracts affected in mild traumatic brain injury.

\begin{tabular}{|ll|}
\hline WM tract & Number of publications \\
\hline Corpus callosum (splenium 8; genu: 8; body: 2; regions not specified: 6) & 24 \\
\hline Internal capsule & 10 \\
\hline Superior longitudinal fasciculus & 10 \\
\hline Corona radiata & 8 \\
\hline Anterior thalamic radiations & 7 \\
\hline Inferior longitudinal fasciculus & 5 \\
\hline Frontal WM & 4 \\
\hline Fornix & 3 \\
\hline Cingulum & 3 \\
\hline Centrum semiovale & 2 \\
\hline Corticospinal tracts & 2 \\
\hline These data are based on 37 publications since 2011. & 2 \\
WM: White matter. & 2 \\
\hline
\end{tabular}




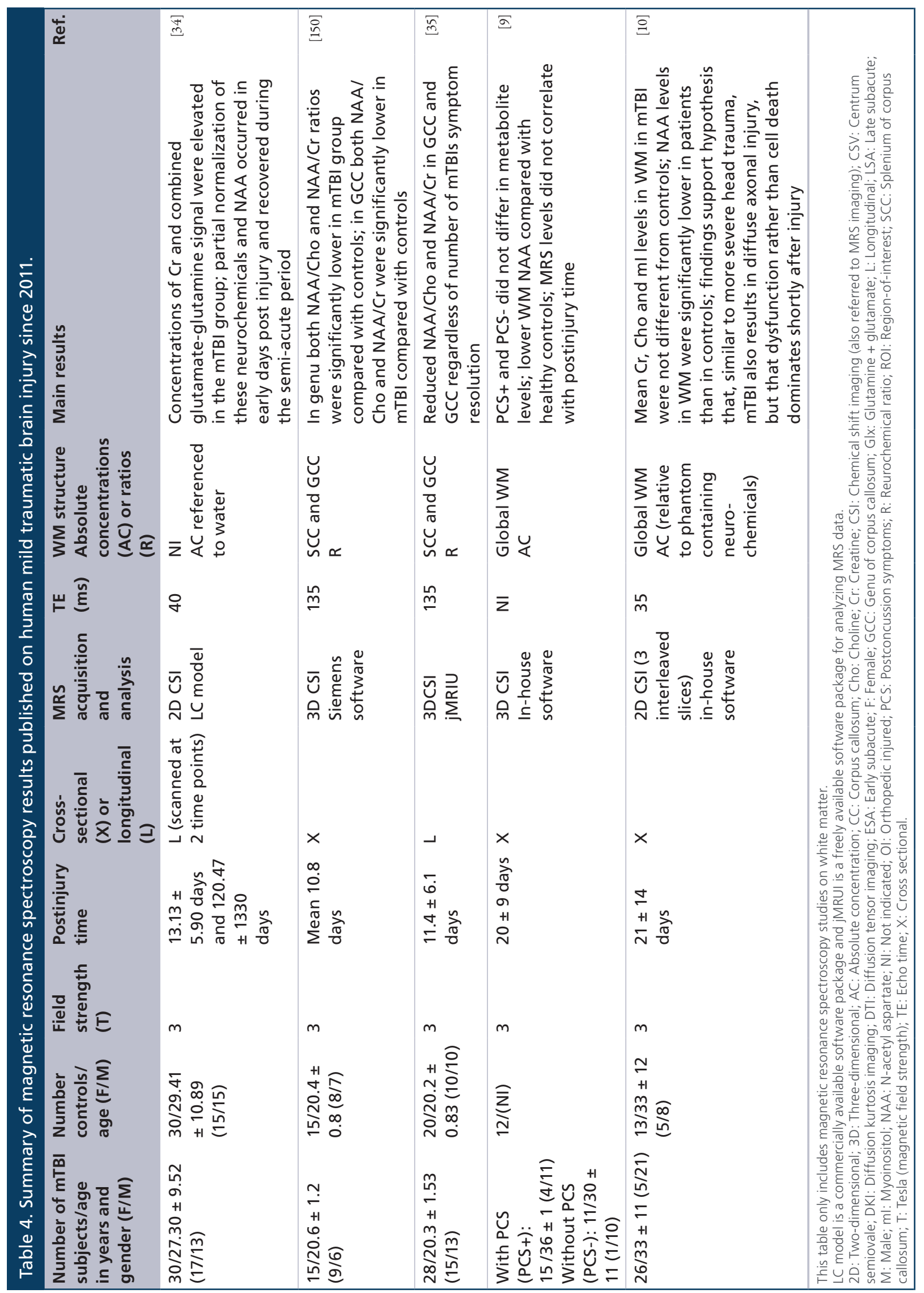




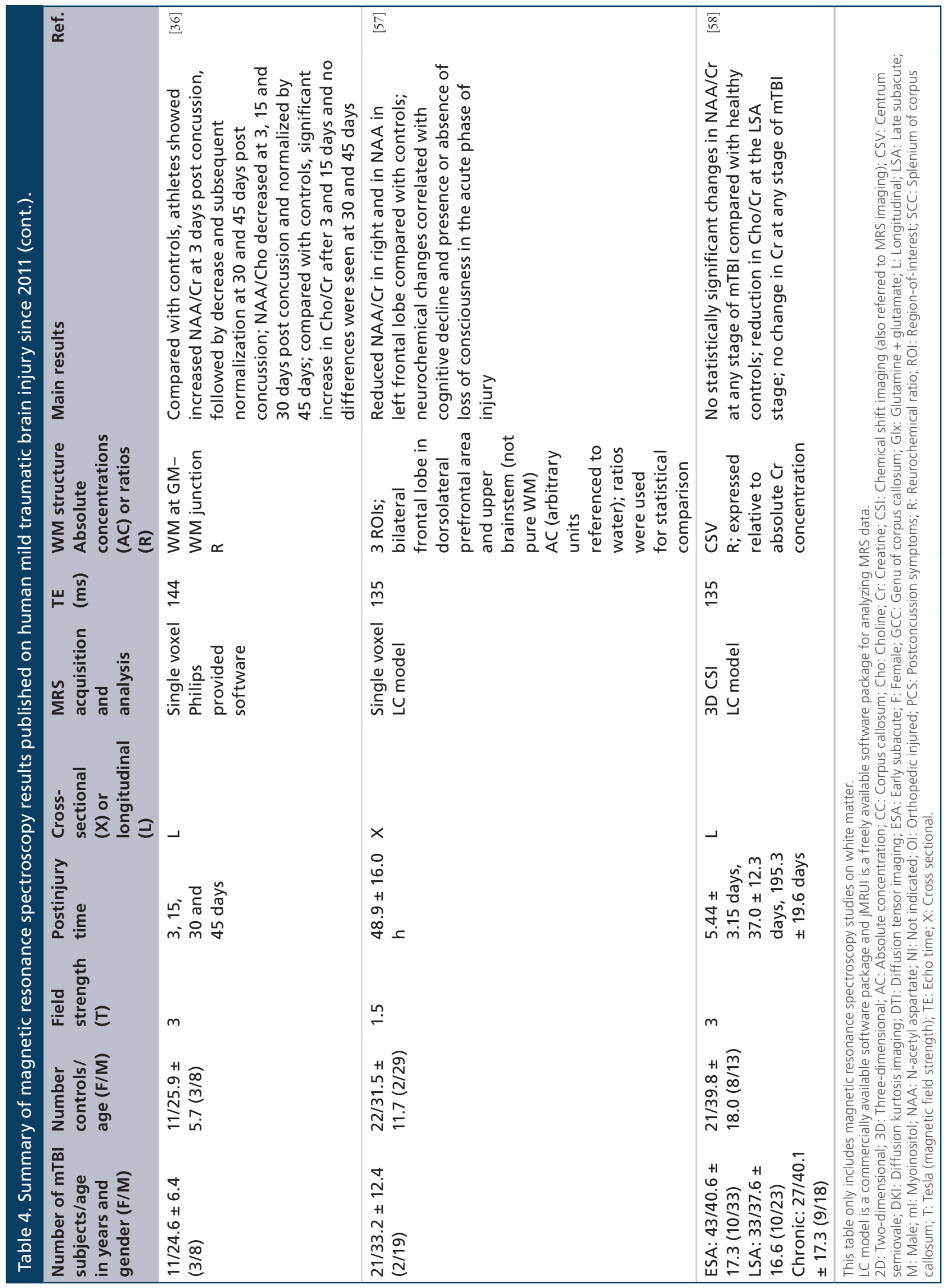




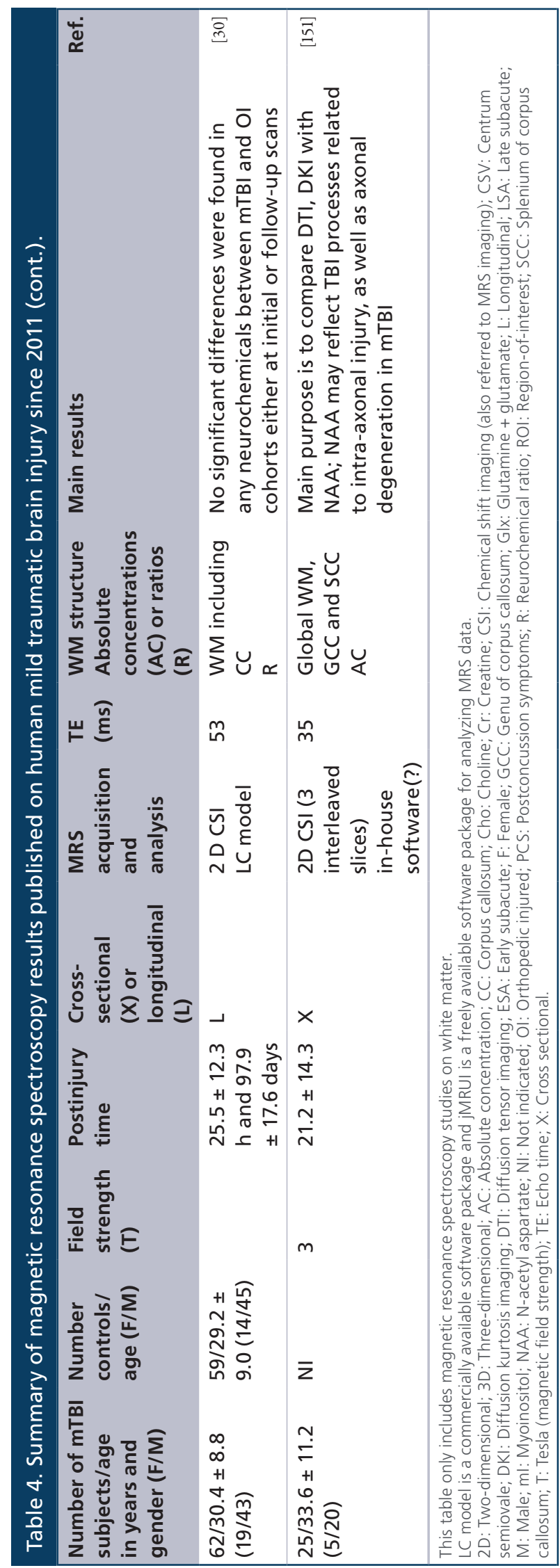

experimental conditions. This limitation can be overcome by rapid and quantitative MTR techniques such as macromolecular proton mapping [118].

\section{Myelin water imaging}

As indicated above, protons associated with myelin are much less mobile and have short T2 short relaxation time that varies from $10 \mu$ s to $1 \mathrm{~ms}$ and are difficult to detect with conventional MRI. However, water protons trapped between the myelin layers have a T2 relaxation value around $30 \mathrm{~ms}$ and can be detected using multi-echo sequences [119]. The myelin water fraction is defined as the ratio of water trapped between the myelin layers to the total tissue water and is a measure of myelin concentration. It is generally thought that this is an indirect measure of myelin.

\section{Ultrashort TE technique}

As indicated above myelin has a very short relaxation time that is difficult to detect using conventional MRI. However, ultrashort echo time (UTE) techniques allow the detection of tissues with very short T2 relaxation times. The UTE techniques have been recently reviewed [120].

\section{Anatomical MRI}

Hyperintense lesions in mTBI are sometimes observed on the T2-weighted FLAIR (fluid attenuation by inversion recovery) images. FLAIR sequence is used to suppress species with long $\mathrm{T} 1$ relaxation times, such as CSF in the brain. This is an excellent sequence for visualizing WM lesions, particularly in the periventricular lesions, in a number of neurological diseases such as multiple sclerosis [121].

Atrophy is generally thought to represent either loss of myelin and/or axons. MRI is an excellent modality for estimating regional, tissue-specific and whole brain atrophy. Atrophy is generally measured on highresolution 3D T1-weighted images which show excellent GM-WM contrast. Cerebral atrophy is useful for following temporal changes in disease progression and response to treatment in neurological disorders such as multiple sclerosis [121] and Alzheimer's disease [122,123].

\section{Magnetic resonance spectroscopy}

Magnetic resonance spectroscopy (MRS) exploits small differences in the nuclear resonance frequency as a result of the local chemical environment. These small frequency differences allow the detection and quantification of various molecules. Since biochemical changes precede anatomical changes, MRS has the potential to detect tissue pathology prior to visualizing on anatomical imaging. The four biologically most important nuclei for MRS are ${ }^{1} \mathrm{H}$ (proton), ${ }^{13} \mathrm{C},{ }^{23} \mathrm{Na}$ 
and ${ }^{31} \mathrm{P}$. Of these nuclei, ${ }^{1} \mathrm{H}$ has the largest abundance in tissue and highest MR sensitivity. In addition, ${ }^{1} \mathrm{H}$ MRS (PMRS or simply, MRS) does not require additional hardware. Therefore, majority of the published studies focused on PMRS.

The major neurochemicals that can be detected with MRS are: $\mathrm{n}$-acetylaspartate, (NAA) + N-acetylaspartylglutamate (NAAG), total creatine $(\mathrm{Cr}$; creatine + phosphocreatine), total choline (Cho; has contributions from multiple molecules that include phosphorylcholine, glycerophosphorylcholine and choline plasmalogen, and a minor contribution from acetylcholine and choline), myoinositol (mI) and lipids. The resonant frequencies of NAA and NAAG are very close and cannot be easily separated. And also the concentration of NAAG is much lower than NAA. Therefore, in the literature, the combined peak is generally referred to as NAA. In addition to the above molecules, one can also detect lactate when it is present. Glutamate and glutamine overlap and difficult to resolve clearly at clinically used magnetic field strengths and the combined peak is referred to as Glx. Note that Glx may also have contributions from gamma-aminobutyric acid (GABA). Detection and quantification of molecules such as GAB require some type of editing. The MRS peaks are identified by their spectral location which is most commonly expressed as parts per million (ppm) and is independent of the magnetic field strength. For example, the resonance frequencies of NAA, $\mathrm{Cr}$ and Cho are 2.02, 3.0 and $3.2 \mathrm{ppm}$, respectively. Of all these neurochemicals, NAA perhaps attracted the greatest attention because it is considered to be a neuronal (cell bodies, axons and dendrites) marker in adult brain. Creatine is an indicator of total energy and intracellular metabolism. It is generally thought that $\mathrm{Cr}$ is unaffected by tissue pathology and is often used as an internal reference. However, a number of studies have shown that the level of $\mathrm{Cr}$ is affected by pathology. Choline reflects cell membrane metabolism. Elevated choline is a marker of cellular turnover and cellular proliferation. Myoinositol is considered to be a glial marker and precursor of phospholipid membrane constituents and its concentration is affected by the formation and breakdown of myelin. The concentration of lactate is fairly low in normal brain (CSF has a higher concentration of lactate than the brain parenchyma) and elevated lactate is a marker of anaerobic glycolysis.

MRS can be acquired either from one ROI at a time (referred to as single voxel MRS or SVS) or simultaneously from multiple voxels, referred to as MRS imaging (MRSI) or chemical shift imaging (CSI). SVS is simple to acquire and generally has superior MRS quality. MRSI allows visualization of spatial distribution within a large region and is technically a little more demanding than SVS and requires longer acquisition times. On modern MRI scanners both SVS and MRSI can be acquired routinely. For technical reasons, most frequently MRSI is acquired from a limited region in the brain. This requires spatial localization and the two most common sequences for spatial localization are point-resolved spectroscopy (PRESS) and stimulated echo acquisition mode (STEAM). The SNR with PRESS is twice that of STEAM. But STEAM can be acquired at shorter echo times than PRESS, thus allowing recovery of some of the lost SNR. It is also possible to acquire MRS from the whole brain without any localization, but is technically challenging. Since the neurochemical concentrations are several orders smaller than tissue water, techniques for water peak suppression are most commonly employed in MRS. However, more recently proton MRS techniques without water suppression are introduced [124].

MRS can be acquired either at short echo times (TE; $\sim 30 \mathrm{~ms}$ or less) and long echo times ( $\sim 140$ or $\sim 270 \mathrm{~ms})$. The advantage of short echo MRS is that neuochemicals with short $\mathrm{T} 2$ relaxation times such as $\mathrm{mI}$ and Glx can be visualized. However, not all the spectral peaks are resolved at short echo times, making it difficult to quantify. The disadvantage of long TE MRS is the difficulty in visualizing neurochemicals with short T2 relaxation times. However, in long TE MRS, the peaks show reduced overlap and the baseline is cleaner for more robust quantification. Long TE MRS is most commonly used to reduce the contamination from lipids (which have a shorter T2 relaxation times) and visualize lactate which overlaps with lipid peaks.

The two major issues with MRS are the relatively poor spatial resolution and long acquisition times. This is one reason why MRS has not gained popularity in routine clinical studies even though it predates MRI.

MRS is inherently a quantitative technique. The simplest way to quantify neurochemical concentrations is to express them as ratios relative to Cr. This assumes that $\mathrm{Cr}$ is unaffected by pathology which is not always true. There are number of software packages, both commercial and free for spectral quantification and determination of both absolute concentrations and as ratios. More details can be found in two recent reviews $[125,126]$.

\section{Application of MRI to WM injury in $\mathrm{mTBI}$}

The application of the above-described MRI techniques to investigate WM changes in $\mathrm{mTBI}$ is reviewed below.

\section{DTI}

The changes in WM on DTI are mainly assessed based on the altered FA and MD values relative to some con- 


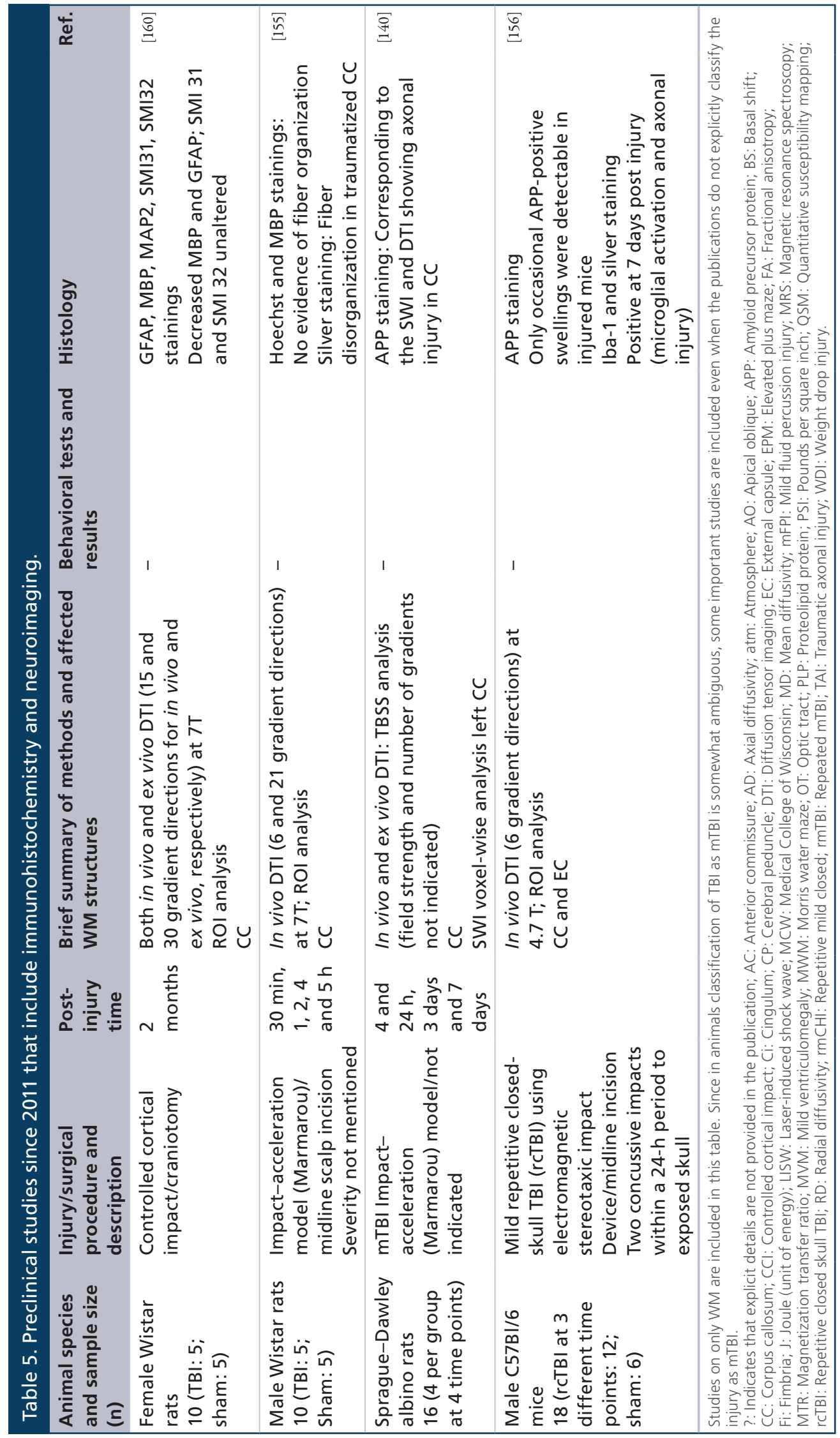




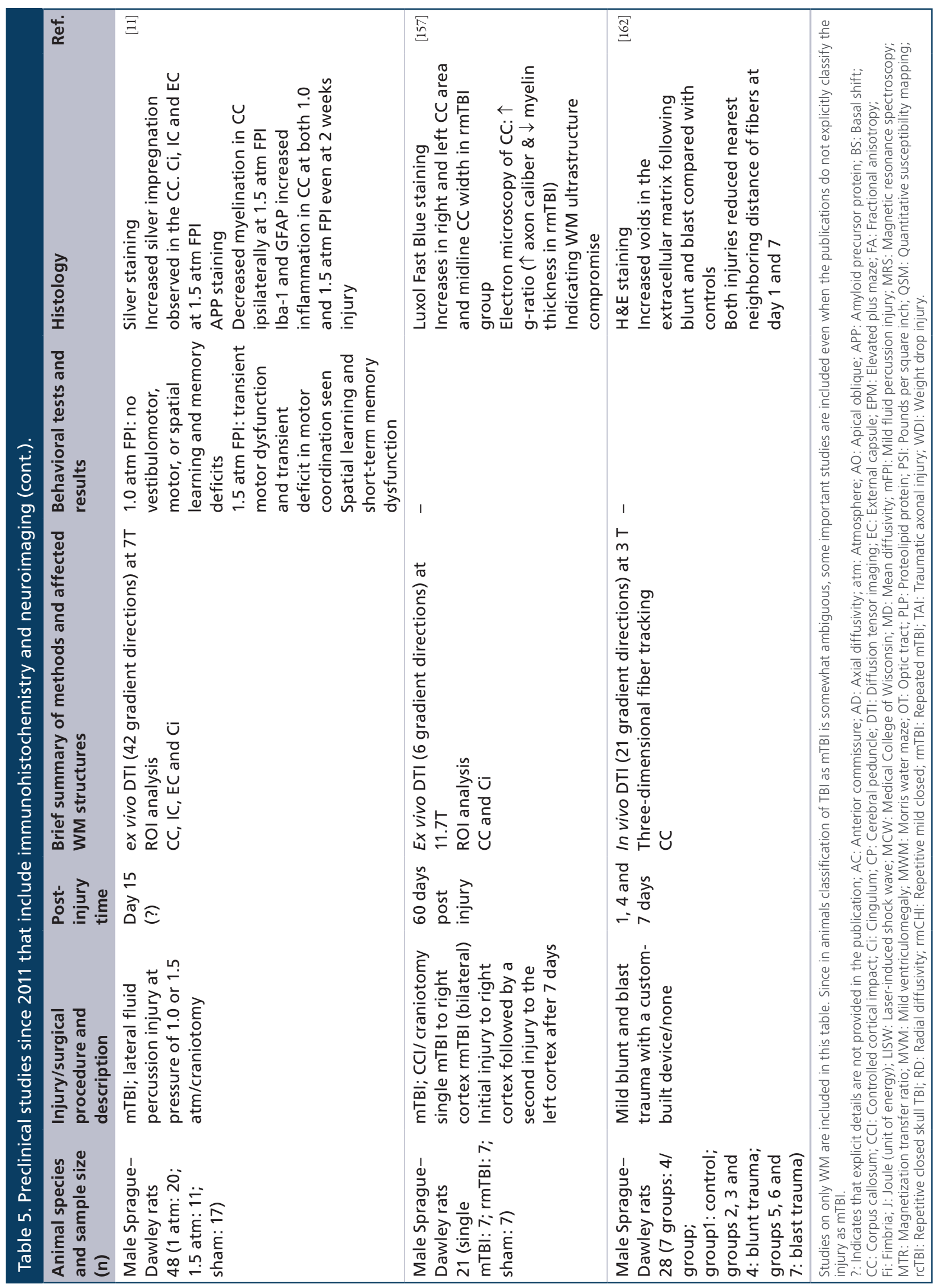




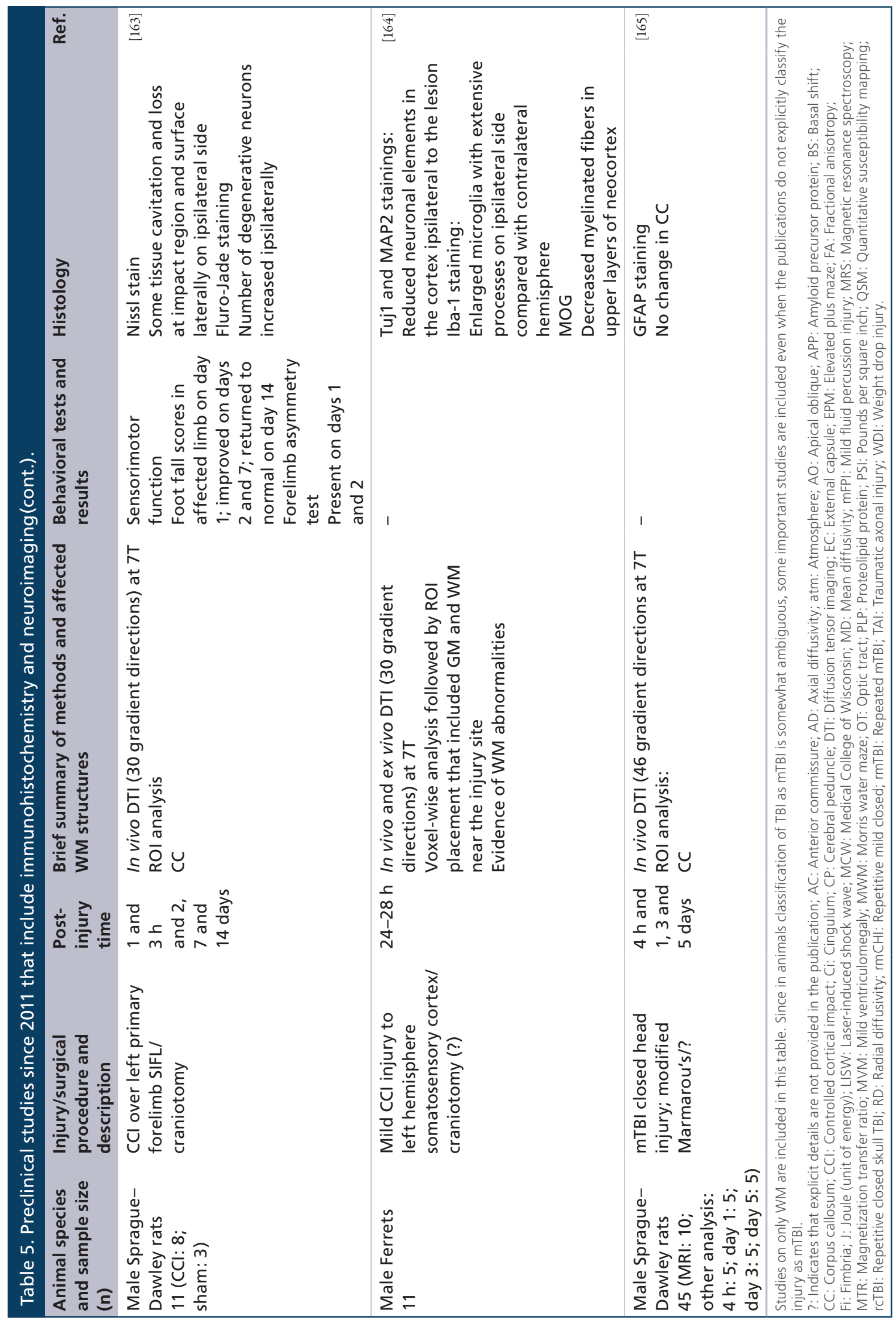




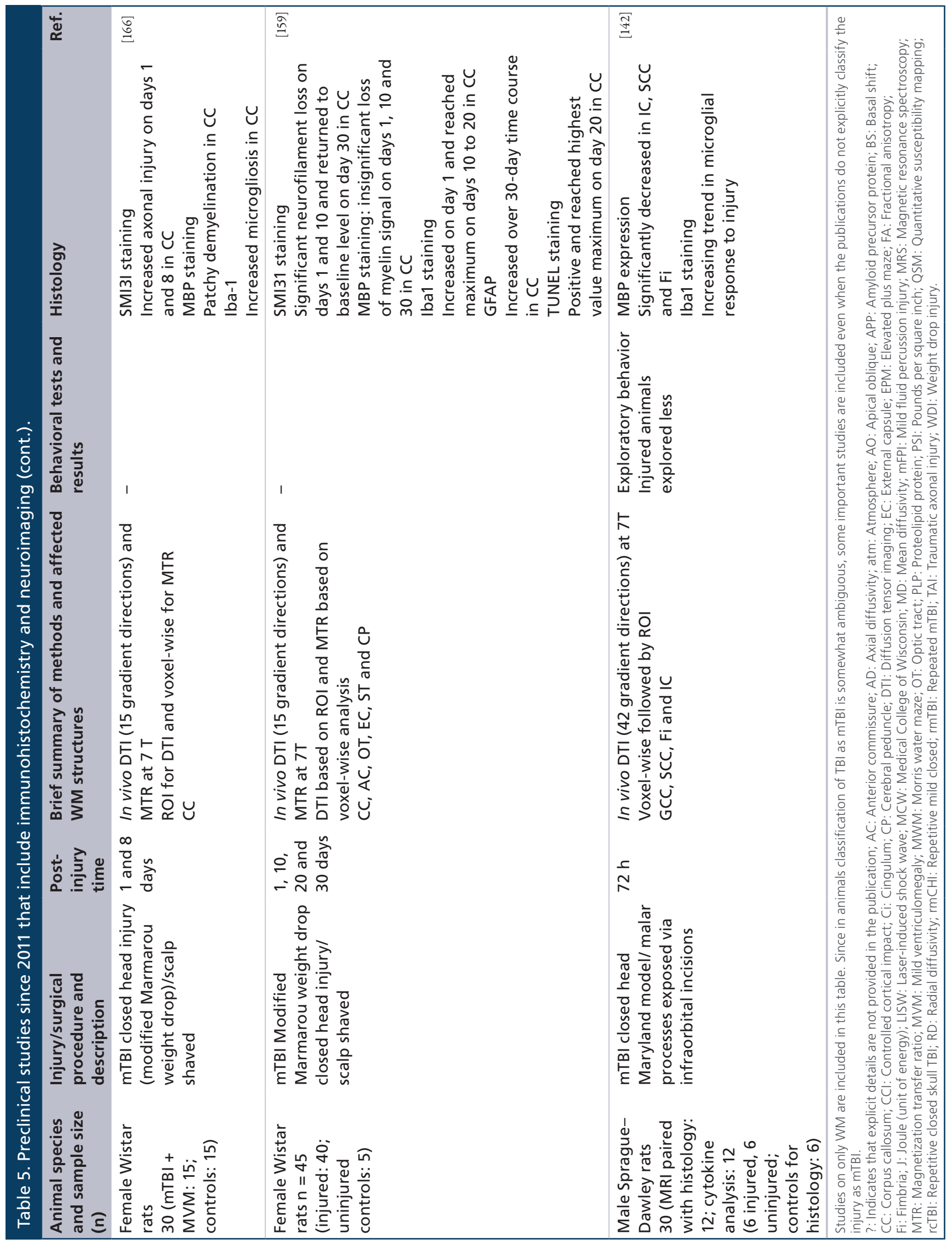




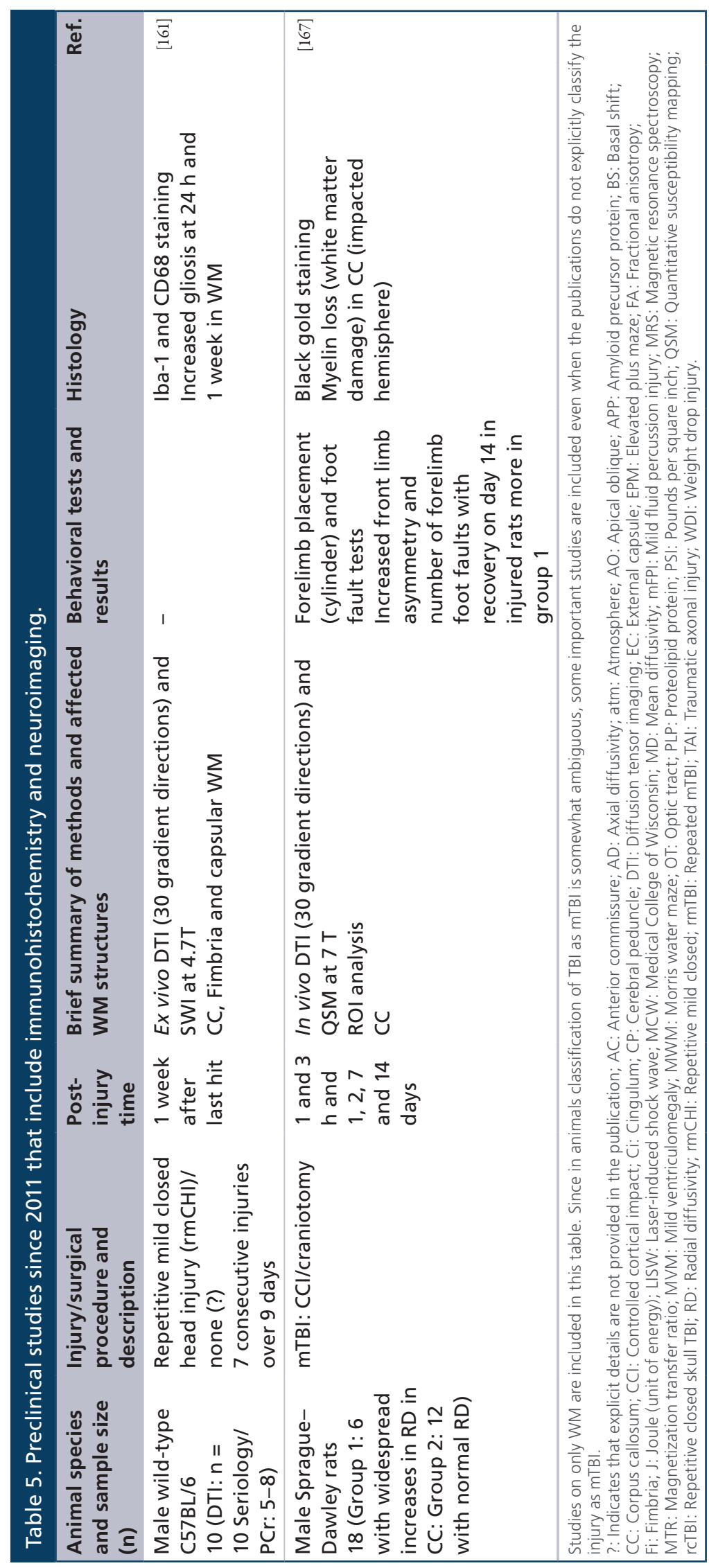

trol group or baseline values. Table 3 summarizes the published literature on the number of affected tracts in mTBI. This table is compiled based on 37 publications after 2011. As can be seen from this table, there is a lack of complete agreement on the location and number of affected WM tracts across different studies. For example, 24 out of 37 publications reported CC to be affected. This is followed by the IC; 10/37, superior longitudinal fasciculus (SLF; 10/37), CR (8/37) and anterior thalamic radiations (ATR; 7/37).

A meta-analysis of the data based on 13 DTI studies (1980 through April 2012) based on the ROI analysis indicated that CC, particularly the splenium (SCC), was the most commonly affected WM structure in mTBI [77]. Another review of the published studies on sports-related concussion through February 2012 identified CC, IC, SLF as the structures most affected in mTBI [78]. It should be noted that the latter analysis is based on only eight studies. Another more recent metaanalysis of the voxel-based data generated in 17 studies (covering until 10 August 2015) determined that the SCC, ATR, left forceps minor and right SLF were the WM structures that were most commonly affected [91].

Some of the reasons for this discordance across different studies could be due to differences in the type of injury, DTI acquisition and analysis methods, post injury scan time, gender, sample sizes, use of improper comparison group and possible hemispheric asymmetry [30,60,63,107,127-129]. In addition, not all studies examined all of the WM structures. For example studies using ROI approach may have examined only a few selected tracts. As indicated earlier, the DTI results strongly depend on the post injury acquisition time [50]. For example, increased RD and reduced FA were observed within 72-h post injury that recovered after 2 weeks [59]. Similarly, changes in some DTI measures were observed around $24 \mathrm{~h}$ post injury time that were normalized by 3 months [30,61].

There is also disagreement on the direction of changes (increase or decrease) in the DTI measures in mTBI relative to baseline or the control group. Majority of the studies on mTBI reported lower FA and higher MD in WM [29]. Other studies reported decreased FA and increased MD at later postinjury time points. In contrast, other studies found increased FA even at later postinjury time points [7]. A metaanalysis revealed higher FA and lower MD in the acute phase, but opposite trend in the chronic phase [80]. In contrast, as reviewed elsewhere, some studies did not show differences in the DTI parameters at any postinjury time point [26]. Another meta-analysis of DTI data in the semi-acute phase of injury revealed that approximately equal number of studies reported elevated and reduced FA values in mTBI [107]. While 
increased FA and reduced MD are thought to represent cytotoxic edema, this interpretation was challenged [7]. As can be seen from Table 1, there is a large spread in the post injury times across different published studies. The effect of these various factors on the reported results makes an objective comparison of DTI results across different studies difficult. Despite the limited agreement across different studies, there is a general consensus that DTI is the most sensitive neuroimaging modality for detecting differences between $\mathrm{mTBI}$ and controls at a group level.

\section{Is DTI ready for managing individual patients?}

Published studies clearly demonstrate considerable intersubject variations in FA that might reflect differences in anatomy, vulnerability to injury and injury mechanisms, among others $[7,29,46,54,55,75]$. Group analyses tend to mask differences among individual subjects. Detecting group differences in DTI parameters, while important, may be of little use for managing individual patients [26]. The TRACK-TBI study also pointed out the importance of considering individual subjects for predicting outcome in $\mathrm{mTBI}$ [42]. We are aware of only three publications that analyzed the data at an individual patient level $[7,52,54]$. For DTI to play an important role in patient management, it is critical to demonstrate the sensitivity of DTI in detecting changes in individual subjects. Using EZ-MAP approach that is shown to be robust for analyzing individual subject's data [54], Lipton et al. [7] reported 'unique spatial patterns' that are 'attributable to interindividual differences in anatomy, vulnerability to injury and mechanism of injury'. Boiux et al. [52] reported that in patients with persistent post concussive symptoms, the individualized analysis has the potential for improving clinical management of these patients.

\section{Advanced diffusion imaging}

The application of DKI to mTBI was recently reviewed [92]. So far only a limited number of studies used DKI for investigating WM changes in mTBI. For example using DKI, an association between mean kurtosis in various WM structures and attention, concentration, memory, learning and information processing was reported [130]. DKI was also reported be sensitive for following pathophysiological changes in the anterior and posterior capsule areas [131]. These authors also computed the DTI measures from the same data by dropping the terms containing higher order b-values. Their studies suggest that RD, AD, MD and FA may underestimate the extent of injury. However incorporating both DTI and DKI measures may improve assessment of tissue microstructure.

As indicated earlier, a single tensor model, by ignor- ing crossing and kissing fibers, may lead to misinterpretation of the results, but also may underestimate the extent of WM injury. Indeed a study using HARDI with 55 diffusion-gradient directions found WM damage to be more widely distributed than previously reported with DTI in Iraq and Afghanistan veterans with previous history of mTBI [132].

Injury to WM along the inter- and/or intrahemispheric pathways can disrupt brain connectivity [133]. Therefore, WM injury in mTBI was also investigated by probing the connectivity between different brain regions. The connectivity can be probed by combining DTI and resting state functional MRI (rfMRI; see [88] for a review). For example, functional connectivity between the two hemispheres in asymptotic mTBI subjects was reported to be reduced that may affect the structural integrity of the CC [134]. Altered structural and functional connectivity within the emotional and perception networks in the acute stage of mTBI was also observed [66]. Using machine learning, altered connectivity within inter- and intrahemispheric WM pathways that may be associated with diffuse axonal injury was demonstrated [135,136]. It is clear from this brief description that connectivity studies have the potential to yield a wealth of information on subtle WM injury that may not otherwise be apparent.

\section{Susceptibility weighted imaging}

Microhemorrhages in WM may indicate DAI [8]. Microhemorrhagic lesions in the SCC and number of GM structures were observed at 6 months post injury on SWI [137]. In contrast, a prospective pilot study at $3 \mathrm{~T}$ on college hockey players failed to detect microhemorrhages at $72 \mathrm{~h}, 2$ weeks and 2 months after concussion [73]. In another study only about 9.8\% amateur boxers showed more hemorrhagic lesions than controls on SWI [138]. However, this study was performed at $1.5 \mathrm{~T}$ and it is possible that more hemorrhagic lesions could have been detected at higher field strengths. Overall, there is no complete agreement on the presence of microhemorrhagic lesions in mTBI.

The relationship between microhemorrhagic lesions and behavioral and cognitive deficits was investigated only in a few studies. A negative correlation between the microhemorrhagic lesions on SWI and cognitive impairment was reported [137]. However, it is not clear if this correlation is the result of hemorrhagic lesions in the SCC or GM. The multicenter TRACK-TBI study on 135 mTBI subjects showed that four hemorrhagic lesions early on after injury was predictive of outcome at 3 months [139]. However, these authors did not explicitly state if these are WM or GM lesions or a combination of both.

The total SWI lesion volume is suggested to be an 
indicator of injury severity [140]. While the SWI lesion volume could reflect injury severity, care should be exercised in relating the number of hemorrhagic lesions to the injury severity since the microbleeds could expand with time and become confluent that results in reduced number of bleeds [141]. Overall there is dearth of literature on WM SWI lesions in mTBI. Thus it is difficult to draw robust conclusions about the role of SWI lesions in WM injury.

\section{Magnetization transfer imaging}

MTR is thought to be an excellent modality for probing myelin integrity. Based on both human and animal studies, myelin appears to be compromised in the initial phase of injury followed by recovery [97,142-144] . Demyelination compromises trophic support to axons and also releases neurite outgrowth inhibitor, Nogo, which has a negative effect on axonal growth and plasticity [97]. Despite its potentially important role, application of MTR to mTBI is limited. Earlier studies reported MTR changes in mTBI [38]. A recent prospective longitudinal study did not show MTR differences between mTBI and orthopedic controls either at approximately $24 \mathrm{~h}$ or approximately 3 months post injury time [30]. It is possible that $24 \mathrm{~h}$ is too early to observe changes in myelin and complete remyelination may have occurred by 3 months, explaining these negative results. Indeed a prospective study using myelin-water imaging of university hockey players reported decreased myelin water fraction at 2 weeks post injury that returned to the preseason level by 2 months after injury [67].

As indicated earlier, macromolecular proton fraction (MPF), a technique based on MTR, was used to investigate veterans who experienced blast mTBI [55]. This study indicated reduced MPF in CC, IC, SLF, superior parietal lobule and right precuneus compared with nonblast veterans. In addition, reduced MPF was also observed at the GM-WM junction that is consistent with DAI. It is however, unclear if reduced MPF completely reflects compromised myelin.

\section{Myelin water imaging}

In spite of the ability of myelin water imaging to directly map the myelin water fraction, to the best of our knowledge, there is only a single study that applied this technique to concussion [67]. In this prospective and longitudinal study on two college hockey teams, reduced myelin water fraction was observed in multiple WM regions that included SCC, CR, right posterior thalamic radiation, left superior CR, left SLFC and left posterior limb of the IC at 2 weeks post injury followed by recovery by 2 months, suggesting transient myelin disruption. This study did not evaluate the behavioral changes in these players.

\section{Ultrashort TE imaging}

UTE is capable of detecting macromolecules such as myelin with short T2 relaxation times. However, to the best of our knowledge, this technique so far has not been applied to mTBI.

\section{Atrophy}

The number of published studies on WM atrophy is relatively small. Majority of the studies focused on whole brain atrophy and GM structures (see recent review [38]). WM atrophy was reported in the anterior cingulate WM and left cingulate gyrus isthmus WM [145]. In this study 28 mTBI patients and 22 controls were followed up to 1 year. These authors reported a correlation between atrophy of the left and right rostral anterior cingulum with changes in memory. They also reported a correlation between the left cingulate gyrus isthmus WM volume with PCS. WM atrophy in mTBI was also reported by others $[146,147]$. However, a longitudinal prospective study, using sophisticated tensor based morphometry, failed to observe atrophy in mTBI either at $24 \mathrm{~h}$ or 3 months relative to orthopedic injured controls [30]. It is possible that 3 months is too a short time to observe regional or global atrophy. Clearly longitudinal studies over a longer period of time are needed to assess the role of WM atrophy in $\mathrm{mTBI}$.

\section{FLAIR}

WM hyperintense lesions on FLAIR sequence were observed in mTBI [32]. In veterans with $\mathrm{mTBI}$, it was shown that these lesions have an effect on verbal memory, independent of the presence of PTSD [148]. Unlike moderate and severe TBI, FLAIR hyperintense lesions are not that common in mTBI. When present, they are very nonspecific, and their significance is not known (perhaps they represent small vessel disease or residual shear-strain injury to WM). Further, it is not clear that these lesions are the result of mTBI or represent pre-existing condition.

\section{Magnetic resonance spectroscopy}

MRS is second only to DTI as the most commonly used MRI-based technique in mTBI. Published MRS studies since 2011 are summarized in Table 4. This table does not include MRS articles that addressed only GM. Nor does this table include any review articles. The application of MRS to $\mathrm{mTBI}$ was reviewed elsewhere [31,38,81,149].

Majority of the MRS studies report reduced NAA in the acute phase of injury $[12,33,36,57]$. A reduction in NAA/Cr and NAA/Cho in the subacute phase of injury in athletes with multiple concussions was also reported [35]. In a longitudinal study reduced NAA and 
elevated Cr and Glx in WM around day 13 post injury in the mTBI group was observed that was followed by partial recovery during the semi-acute period [34]. This study also noted that premorbid intelligence predicts the degree of recovery of the neurochemicals indicating that the degree of recovery depends on the biological factors underlying intelligence. In a cross-sectional study on concussive injured athletes continued neurochemical alterations were observed even when the clinical symptoms have resolved [152]. This is consistent with other reports using advanced neuroimaging techniques $[33,88,153,154]$. In another study in acute phase neurochemical changes were observed only in subjects with LOC [57]. However, it appears that the ROI's in this study represent a mixture of both GM and WM and therefore the reported association may not be attributed completely to WM injury. In an interesting study on a small number of subjects, the WM levels of NAA, Cr, Cho and mI were compared in mTBI subjects with and without PCS [9]. These authors reported changes in these neurochemicals levels in PCS positive, but not in PCS negative subjects. This suggests that MRS has the potential to differentiate between PCS positive and PCS negative subjects. In another study, the regional metabolic levels were correlated with neuropsychological data [58]. These authors also investigated if neurochemical changes observed in the early subacute phase (within 10 days post injury time as defined by these authors) were predictive of neurocognitive outcome at 6 months post injury. This study demonstrated an association between Cr levels in the centrum semiovale in the early subacute phase and the automated neuropsychological assessment metrics scores in the chronic phase. In addition, these authors also reported decreased $\mathrm{Cho} / \mathrm{Cr}$ in the late subacute phase. These results suggest that MRS measures have the potential to serve as diagnostic and prognostic markers in mTBI. Consistent with other studies, the neurochemical levels returned to the normative values in the later stage of injury.

There is no complete agreement about the changes in the neurochemical levels in mTBI. For example a few studies failed to observe any changes in NAA levels $[30,34,152]$. A prospective study on an mTBI cohort failed to observe changes in NAA/Cr, Cho/Cr and $\mathrm{mI} / \mathrm{Cr}$ either, at approximately $24 \mathrm{~h}$ or approximately 3 months post injury [30]. Based on the published literature, it is likely that $24 \mathrm{~h}$ post injury is too early for metabolic changes to occur and any possible neurochemical changes may have disappeared by 3 months [36].

A review of Table 4 explains why it is very difficult to generalize the conclusions reported across different MRS studies. Many studies did not specify the WM regions from which the spectra were acquired even though it is known that metabolite levels depend on the brain area (see e.g. [45,81]. The type of MRS acquisition varied across different studies. Some studies employed single voxel technique while others used 2D and 3D MRSI using different echo times. A few studies expressed the neurochemicals as ratios while others expressed as absolute concentrations. Ratios would not allow assessment of whether the altered numerator or denominator is responsible for the observed neurochemical changes. For example, many of the conclusions about NAA reduction are based on measured $\mathrm{NAA} / \mathrm{Cr}$ ratios. This assumes that the $\mathrm{Cr}$ levels are not affected by $\mathrm{mTBI}$, which is a questionable assumption. A few studies that reported absolute concentrations used a variety of methods to quantify neurochemicals. The MRS data were acquired at different postinjury times even though the neurochemical levels were shown to change with postinjury time [10,58]. Another problem is the placement of the ROIs. Because of the relatively poor spatial resolution, many of the ROIs included part of GM that makes it difficult to attribute the observed changes only to WM pathology. Finally majority of the studies were based on a relatively small sample size.

\section{MRI \& pathology correlation}

Histology is the gold standard for evaluating mTBIinduced pathology. However, histologic evaluation is not ethically justified in human mTBI. This is where noninvasive neuroimaging such as MRI could play a central role. While MRI is an excellent radiologic modality for visualizing and following pathology, its pathologic specificity is somewhat limited. In mTBI the commonly observed MRI abnormalities in WM are: altered diffusion measures that include FA, RD, $\mathrm{AD}, \mathrm{MD}$ on DTI; hypointense lesions on SWI; atrophy (seen on anatomical MRI); reduced MTR on MTI; changes in the myelin water fraction on myelin water imaging and macromolecular fraction imaging; and altered neurochemical concentrations (NAA, Cr, Cho and $\mathrm{mI}$ ) on MRS. In the absence of histologic confirmation, our interpretation of the MRI changes is mainly based on preclinical models of mTBI. Since DTI is the most commonly used imaging modality in mTBI, majority of the imaging-pathology correlation studies focused on DTI.

There is significant literature on the MRI-pathology correlation in different diseases. However this correlation may depend on the disease type. There are only a handful of studies that investigated the MRI-histology correlations in preclinical models of mTBI. A summary of the published MRI-histology correlation studies is provided in Table 5. Almost all the published studies agree that various pathological substrates contribute 
to the observed changes in the DTI parameters and establishing one-to-correspondence between the DTI measures and histology is not straight forward. Majority of the studies suggest that axonal injury (including demyelination) mainly contributes to the observed alterations in the DTI measures [11,137,141,155-159]. However, some authors argue that inflammation also contributes to the altered DTI measures $[160,161]$. There is some agreement that $\mathrm{RD}$ is a measure of myelin integrity $[142,157,159]$. AD is thought to represent axonal damage [155]. However, there is some disagreement about this interpretation $[142,160,161]$. In summary, based on the published studies it is not straightforward to relate changes in DTI measures to pathology since they are affected by coherence of the fiber tracts, inflammation, gliosis, demyelination and axonal compromise [145].

The lesions seen on SWI are shown to correlate with hemorrhage on histology [140,161]. However, care must be exercised in interpreting the SWI lesions since it is sometimes difficult to directly compare the Prussian reaction, used for identifying hemorrhage, with heme degradation [141,161].

MTR is generally thought to reflect the state of myelin. Based on a recent study, MTR appears to be more affected by astrogliosis and microgliosis than demyelination [159]. MTR changes also depend on the offset frequency of the off-resonance pulse [159]. For example, the MTR with off-resonance frequency of $3.5 \mathrm{ppm}$ reflects both axonal and myelin integrity while MTR with offset frequency of $20 \mathrm{ppm}$ mainly reflects astrogliosis. Thus it appears difficult to attribute MTR changes to a single pathologic component.

To the best of our knowledge there are no studies that reported correlation between MRS, MWF with pathology in mTBI.

As can be seen from this brief summary of the MRI-pathology correlative studies, MRI measures reflect different pathologic components, depending on the time and type of injury, experimental conditions. Robust correlation, between MRI findings and neuropathology requires the use of appropriate animal models of mTBI. However, there is a lack of consensus on what represents mTBI in animal models [168-171]. The commonly used neurological severity score that is a composite of behavioral and motor functions and righting reflexes for grading severity of TBI $[168,172]$ is of limited use in in experimental models of mTBI. Even with all these limitations, DTI, MRS, MTR and SWI measures are very sensitive imaging modalities for detecting mTBI-induced pathology.

\section{Conclusion \& future perspective}

Based on the above review it is clear that the multimodal neuroimaging has diagnostic and prognostic values in detecting and evaluating WM changes in mTBI. These MRI-based modalities are also able to detect ongoing pathology even when the subjects are asymptomatic. However, MRI measures also show wide variation across different studies. In order to properly compare results from different studies to develop a coherent picture about the MRI-detected pathology and its correlation with cognitive and behavioral deficits, it is essential to standardize the acquisition and analysis methods. Similarly there is a need to standardize the behavioral and cognitive measures. Majority of the publications are based on cross-sectional studies with relatively small sample size. Cross-sectional studies have lower statistical power relative to longitudinal studies. Longitudinal studies also provide important information about the evolution of pathology. For example we know relatively little about the long-term consequences of mTBI. Given the heterogeneity of the injury, age, gender and other variables, studies based on a small cohort may not allow for robust conclusions. This requires studies on large and well-characterized cohorts. However, it may not be easy to recruit adequate number of subjects needed for statistically robust results by a single center. Recruiting large cohorts requires multicenter design with well-standardized protocol and rigorous quality control in place. It is also important to include both genders since some of the published studies show gender effect on pathology and behavioral deficits. It is equally important to consider age as a covariate in the analysis of the results since many of the MRI measures are age dependent. There are a few studies that demonstrate hemispheric asymmetry in mTBI. Future studies should explicitly state the side of the hemisphere in their results. A major problem in the field of $\mathrm{mTBI}$ is the lack of appropriate diagnostic criteria and the absence of a biomarker. As pointed out by Manley and Maas [1], there is a critical need to incorporate imaging information for diagnosing mTBI. Finally there is a need to develop preclinical mTBI models, particularly using large animals, to interpret the observed changes in MRI measures on a rational basis.

\section{Limitations of this review}

This review is based on publications after 2011 and includes only references published after 2011. Thus original references are not cited. However, references to recent reviews are provided, whenever possible, so that the readers can have information about the original work. While there are multiple neuroimaging modalities other than MRI, for this review which focuses on only WM injury, other imaging modalities such as CT, PET, single-photon emission computed tomography, near-infrared are of limited use. Finally, this study did 
not try to separate blast from mechanical mTBI. This study also did not separate single and repetitive mTBIs.

\section{Acknowledgements}

The author thanks Dr. S Kondraganti for her help with the literature search.

\section{Financial \& competing interests disclosure}

The MRI scanners used to acquire MRI data that are included in this review were partially funded by NIH (Grant \# S10 RR19186-01 and S10 RR17205-01). The author has no other relevant affiliations or financial involvement with any organization or entity with a financial interest in or financial conflict with the subject matter or materials discussed in the manuscript apart from those disclosed.

No writing assistance was utilized in the production of this manuscript.

\section{Open access}

This work is licensed under the Creative Commons Attribution 4.0 License. To view a copy of this license, visit http://creativecommons.org/licenses/by/4.0/

\section{Executive summary}

- Noninvasive neuroimaging techniques, particularly MRI-based diffusion tensor imaging and magnetic resonance spectroscopy, have considerably improved our understanding about WM injury in mild traumatic brain injury (mTBI). They also enhanced the diagnosis and prognosis and help follow pathophysiological changes in $\mathrm{mTBI}$.

- Many MRI-derived white matter injury measures correlated with cognitive and neurobehavioral deficits, which suggests a predictive role for this neuroimaging technology.

- There is no complete agreement on neuroimaging results across different studies. A problem with most of the published MRI studies in $\mathrm{MTBI}$ are the variability in injury, postinjury time at which studies are performed and not accounting for the site of impact.

- Most of the published studies are either cross-sectional or are based on a small sample size. There is need for controlled studies on large cohorts.

- Majority of the published studies are based on single center that makes it difficult to validate results across different centers.

- Classification of $\mathrm{mTBI}$ is based on Glasgow Coma Score whose sensitivity is relatively poor in diagnosing mTBI. There is a critical need to incorporate imaging information for diagnosing mTBI.

- Lack of standardization in the acquisition and analysis techniques across centers prevents an objective comparison across different studies.

- While group analysis is important, there is a need to investigate the role of imaging in managing individual patients.

- Imaging-pathology correlation is not always consistent. There is some urgency in performing controlled preclinical studies for a rational interpretation of the MRI results.

- Despite these limitations neuroimaging is indispensable for the management of mTBI.

\section{References}

1 Manley GT, Maas AIR. Traumatic brain injury an international knowledge-based approach. JAMA 310(5), 473-474 (2013).

2 Faul M, Coronado V. Epidemiology of traumatic brain injury. Handb. Clin. Neurol. 127, 3-13 (2015).

3 Hemphill MA, Dauth S, Yu CJ, Dabiri BE, Parker KK. Traumatic brain injury and the neuronal microenvironment: a potential role for neuropathological mechanotransduction. Neuron 85(6), 1177-1192 (2015).

4 Browne KD, Chen XH, Meaney DF, Smith DH. Mild traumatic brain injury and diffuse axonal injury in swine. J. Neurotrauma 28(9), 1747-1755 (2011).

5 Greer JE, McGinn MJ, Povlishock JT. Diffuse traumatic axonal injury in the mouse induces atrophy, c-Jun activation, and axonal outgrowth in the axotomized neuronal population. J. Neurosci. 31(13), 5089-5105 (2011).
6 Sharp DJ, Ham TE. Investigating white matter injury after mild traumatic brain injury. Curr. Opin. Neurol. 24(6), 558-563 (2011).

7 Lipton ML, Kim N, Park YK et al. Robust detection of traumatic axonal injury in individual mild traumatic brain injury patients: intersubject variation, change over time and bidirectional changes in anisotropy. Brain Imaging Behav. 6(2), 329-342 (2012).

8 Bigler ED, Maxwell WL. Neuropathology of mild traumatic brain injury: relationship to neuroimaging findings. Brain Imaging Behav. 6(2), 108-136 (2012).

9 Kirov II, Tal A, Babb JS et al. Proton MR spectroscopy correlates diffuse axonal abnormalities with post-concussive symptoms in mild traumatic brain injury. J. Neurotrauma 30(13), 1200-1204 (2013).

10 Kirov II, Tal A, Babb JS, Lui YW, Grossman RI, Gonen O. Diffuse axonal injury in mild traumatic brain injury: a $3 \mathrm{D}$ multivoxel proton MR spectroscopy study. J. Neurol. 260 (1), 242-252 (2013). 
11 Hylin MJ, Orsi SA, Zhao J et al. Behavioral and histopathological alterations resulting from mild fluid percussion injury. J. Neurotrauma 30 (9), 702-715 (2013).

12 Johnson VE, Stewart W, Smith DH. Axonal pathology in traumatic brain injury. Exp. Neurol. 246, 35-43 (2013).

13 Bayly PV, Clayton EH, Genin GM. Quantitative imaging methods for the development and validation of brain biomechanics models. Annu. Rev. Biomed. Eng. 14, 369-396 (2012).

14 McAllister TW, Ford JC, Ji S et al. Maximum principal strain and strain rate associated with concussion diagnosis correlates with changes in corpus callosum white matter indices. Ann. Biomed. Eng. 40 (1), 127-140 (2012).

15 Ji S, Ghadyani H, Bolander RP. Parametric comparisons of intracranial mechanical responses from three validated finite element models of the human head. Ann. Biomed. Eng. 42(1), 11-24 (2014).

16 Krainin BM, Forsten RD, Kotwal RS, Lutz RH, Guskiewicz KM. Mild traumatic brain injury literature review and proposed changes to classification. J. Spec. Oper. Med. 11(3), 38-47 (2011).

17 Godbolt AK, Cancelliere C, Hincapié CA et al. Systematic review of the risk of dementia and chronic cognitive impairment after mild traumatic brain injury: results of the International Collaboration on Mild Traumatic Brain Injury Prognosis. Arch. Phys. Med. Rehabil. 95(Suppl. 3), S245-S256 (2014).

18 Boyle E, Cancelliere C, Hartvigsen J, Carroll LJ, Holm LW, Cassidy JD. Systematic review of prognosis after mild traumatic brain injury in the military: results of the international collaboration on mild traumatic brain injury prognosis. Arch. Phys. Med. Rehabil. 95(Suppl. 3), S230-237 (2014).

19 Cancelliere C, Hincapié CA, Keightley M et al. Systematic review of prognosis and return to play after sport concussion: results of the international collaboration on mild traumatic brain injury prognosis. Arch. Phys. Med. Rehabil. 95(Suppl. 3), S210-S229 (2014).

20 Messé A, Caplain S, Pélégrini-Issac M et al. Structural integrity and postconcussion syndrome in mild traumatic brain injury patients. Brain Imaging Behav. 6(2), 283-292 (2012).

21 Levin HS, Diaz-Arrasita RR. Diagnosis, prognosis, and clinical management of mild traumatic brain injury. Lancet Neurol. 14(5), 506-517 (2015).

22 McMahon P, Hricik A, Yue JK et al. Symptomatology and functional outcome in mild traumatic brain injury: results from the prospective TRACK-TBI study. J. Neurotrauma 31(1), 26-33 (2014).

23 Clay MB, Glover KL, Lowe DT. Epidemiology of concussion in sport: a literature review. J. Chiropr. Med. 12(4), 230-251 (2013).

24 Williams VB, Danan IJ. A historical perspective on sports concussion: where we have been and where we are going. Curr. Pain Headache Rep. 20(6), 43 (2016).

25 Holtkamp MD, Grimes J, Ling G. Concussion in the military: an evidence-base review of mTBI in US military personnel focused on posttraumatic headache. Curr. Pain Headache Rep. 20(6), 37-43 (2016).

26 Honce JM, Nyberg E, Jones I, Nagae L. Neuroimaging of concussion. Phys. Med. Rehabil. Clin. N. Am. 27(2), 411-428 (2016).

27 Adams RS, Corrigan JOHN D, Larson MJ. Alcohol use after combat-acquired traumatic brain injury: what we know and don't know. J. Soc. Work Pract. Addict. 12, 28-51 (2012).

28 Diaz-Arrastia R, Kochanek PM, Bergold P et al. Pharmacotherapy of traumatic brain injury: state of the science and the road forward: report of the Department of Defense Neurotrauma Pharmacology Workgroup. J. Neurotrauma 31(2), 135-158 (2014).

29 Shenton ME, Hamoda HM, Schneiderman JS et al. A review of magnetic resonance imaging and diffusion tensor imaging findings in mild traumatic brain injury. Brain Imaging Behav. 6(2), 137-192 (2012).

30 Narayana PA, Yu X, Hasan KM et al. Multi-modal MRI of mild traumatic brain injury. Neuroimage Clin. 7, 87-97 (2015).

31 Toth A. Chapter 24: magnetic resonance imaging application in the area of mild and acute traumatic brain injury: implications for diagnostic markers? In: Brain Neurotrauma: Molecular, Neuropsychological, and Rehabilitation Aspects. Kobeissy FH (Ed.). CRC Press/Taylor \& Francis, FL, USA (2015).

32 Bigler ED, Abildskov TJ, Goodrich-Hunsaker NJ et al. Structural neuroimaging findings in mild traumatic brain injury. Sports Med. Arthrosc. Rev. 24(3), e42-e52 (2016).

33 Henry LC, Tremblay S, Leclerc $S$ et al. Metabolic changes in concussed American football players during the acute and chronic post-injury phases. BMC Neurol. 11, 105 (2011).

34 Yeo RA, Gasparovic C, Merideth F, Ruhl D, Doezema D, Mayer AR. A longitudinal proton magnetic resonance spectroscopy study of mild traumatic brain injury. J. Neurotrauma 28(1), 1-11 (2011).

35 Johnson B, Gay M, Zhang K et al. The use of magnetic resonance spectroscopy in the subacute evaluation of athletes recovering from single and multiple mild traumatic brain injury. J. Neurotrauma 29(13), 2297-2304 (2012).

36 Vagnozzi R, Signoretti S, Floris R et al. Decrease in $\mathrm{N}$-acetylaspartate following concussion may be coupled to decrease in creatine. J. Head Trauma Rehabil. 28, 284-292 (2013).

37 Fox WC, Park MS, Belverud S, Klugh A, Rivet D, Tomlin JM. Contemporary imaging of mild TBI: the journey toward diffusion tensor imaging to assess neuronal damage. Neurol. Res. 35(3), 223-232 (2013).

38 Wu X, Kirov II, Gonen O, Ge Y, Grossman RI, Lui YW. MR imaging applications in mild traumatic brain injury: an imaging update. Radiology 279(3), 693-707 (2016).

39 Mayer AR, Bellgowan PS, Hanlon FM. Functional magnetic resonance imaging of mild traumatic brain injury. Neurosci. Biobehav. Rev. 49, 8-18 (2015).

40 Byrnes KR, Wilson CM, Brabazon F et al. FDG-PET imaging in mild traumatic brain injury: a critical review. Front. Neuroenergetics 5, 13 (2014). 
41 Wilde EA, Bouix S, Tate DF et al. Advanced neuroimaging applied to veterans and service personnel with traumatic brain injury: state of the art and potential benefits. Brain Imaging Behav. 9(3), 367-402 (2015).

42 Yuh EL, Cooper SR, Mukherjee P et al. Diffusion tensor imaging for outcome prediction in mild traumatic brain injury: a TRACK-TBI study. J. Neurotrauma 31(17), 1457-1477 (2014).

43 Messé A, Caplain S, Paradot G et al. Diffusion tensor imaging and white matter lesions at the subacute stage in mild traumatic brain injury with persistent neurobehavioral impairment. Hum. Brain Mapp. 32(6), 999-1011 (2011).

44 Lange RT, Iverson GL, Brubacher JR, Mädler B, Heran MK. Diffusion tensor imaging findings are not strongly associated with postconcussional disorder 2 months following mild traumatic brain injury. J. Head Trauma Rehabil. 27(3), 188-198 (2012).

45 Davenport ND, Lim KO, Armstrong MT, Sponheim SR. Diffuse and spatially variable white matter disruptions are associated with blast-related mild traumatic brain injury. Neuroimage 59(3), 2017-2024 (2012).

46 Wilde EA, McCauley SR, Barnes A et al. Serial measurement of memory and diffusion tensor imaging changes within the first week following uncomplicated mild traumatic brain injury. Brain Imaging Behav. 6(2), 319-328 (2012).

47 Wada T, Asano Y, Shinoda J. Decreased fractional anisotropy evaluated using tract-based spatial statistics and correlated with cognitive dysfunction in patients with mild traumatic brain injury in the chronic stage. AJNR Am. J. Neuroradiol. 33(11), 2117-2122 (2012).

48 Rao V, Mielke M, Xu X et al. Diffusion tensor imaging atlasbased analyses in major depression after mild traumatic brain injury. J. Neuropsychiatry Clin. Neurosci. 24(3), 309-315 (2012).

49 Matthews SC, Spadoni AD, Lohr JB, Strigo IA, Simmons AN. Diffusion tensor imaging evidence of white matter disruption associated with loss versus alteration of consciousness in warfighters exposed to combat in operations enduring and Iraqi freedom. Psychiatry Res. 204(2-3), 149-154 (2012).

50 Toth A, Kovacs N, Perlaki G et al. Multi-modal magnetic resonance imaging in the acute and sub-acute phase of mild traumatic brain injury: can we see the difference? J. Neurotrauma 30(1), 2-10 (2013).

51 Grossman EJ, Jensen JH, Babb JS et al. Cognitive impairment in mild traumatic brain injury: a longitudinal diffusional kurtosis and perfusion imaging study. AJNR Am. J. Neuroradiol. 34(5), 951-957 (2013).

52 Bouix S, Pasternak O, Rathi Y, Pelavin PE, Zafonte R, Shenton ME. Increased gray matter diffusion anisotropy in patients with persistent post-concussive symptoms following mild traumatic brain injury. PLoS ONE 8(6), e66205 (2011).

53 Yallampalli R, Wilde EA, Bigler ED et al. Acute white matter differences in the fornix following mild traumatic brain injury using diffusion tensor imaging. J. Neuroimaging 23(2), 224-227 (2013).

54 Kim N, Branch CA, Kim M, Lipton ML. Whole brain approaches for identification of microstructural abnormalities in individual patients: comparison of techniques applied to mild traumatic brain injury. PLoS ONE 8(3), e59382 (2013).

55 Petrie EC, Cross DJ, Yarnykh VL et al. Neuroimaging, behavioral, and psychological sequelae of repetitive combined blast/impact mild traumatic brain injury in Iraq and Afghanistan war veterans. J. Neurotrauma 31(5), 425-436 (2014).

56 Sorg SF, Delano-Wood L, Luc N et al. White matter integrity in veterans with mild traumatic brain injury: associations with executive function and loss of consciousness. Head Trauma Rehabil. 29(1), 21-32 (2014).

57 Sivák Š, Bittšanský M, Grossmann J et al. Clinical correlations of proton magnetic resonance spectroscopy findings in acute phase after mild traumatic brain injury. Brain Inj. 28 (3), 341-346 (2014).

58 George EO, Roys S, Sours C et al. Longitudinal and prognostic evaluation of mild traumatic brain injury: a $1 \mathrm{H}$-magnetic resonance spectroscopy study. J. Neurotrauma 31(11), 1018-1028 (2014).

59 Murugavel M, Cubon V, Putukian M et al. A longitudinal diffusion tensor imaging study assessing white matter fiber tracts after sports-related concussion. J. Neurotrauma 31(22), 1860-1871 (2014).

60 Fakhran S, Yaeger K, Collins M, Alhilali L. Sex differences in white matter abnormalities after mild traumatic brain injury: localization and correlation with outcome. Radiology 272(3), 815-823 (2014).

61 Hasan KM, Wilde EA, Miller ER et al. Serial atlas-based diffusion tensor imaging study of uncomplicated mild traumatic brain injury in adults. J. Neurotrauma 31(5), 466-475 (2014).

62 Alhilali LM, Delic JA, Gumus S, Fakhran S. Evaluation of white matter injury patterns underlying neuropsychiatric symptoms after mild traumatic brain injury. Radiology 277(3), 793-800 (2015).

63 Hayes JP, Miller DR, Lafleche G, Salat DH, Verfaellie M. The nature of white matter abnormalities in blast-related mild traumatic brain injury. Neuroimage Clin. 8, 148-156 (2015).

64 Delano-Wood L, Bangen KJ, Sorg SF4 et al. Brainstem white matter integrity is related to loss of consciousness and postconcussive symptomatology in veterans with chronic mild to moderate traumatic brain injury. Brain Imaging Behav. 9(3), 500-512 (2015).

65 J Babcock L, Yuan W, Leach J, Nash T, Wade S. White matter alterations in youth with acute mild traumatic brain injury. Pediatr. Rehabil. Med. 8(4), 285-296 (2015).

66 Iraji A, Chen $\mathrm{H}, \mathrm{W}$ iseman $\mathrm{N}$ et al. Connectome-scale assessment of structural and functional connectivity in mild traumatic brain injury at the acute stage. Neuroimage Clin. 12, 100-115 (2016).

67 Wright AD, Jarrett M, Vavasour I et al. Myelin water fraction is transiently reduced after a single mild traumatic brain injury--a prospective cohort study in collegiate hockey players. PLoS ONE 11(2), e0150215 (2016).

68 Delic J, Alhilali LM, Hughes MA, Gumus S, Fakhran $S$. White matter injuries in mild traumatic brain injury 
and posttraumatic migraines: diffusion entropy analysis. Radiology 279(3), 859-866 (2016).

69 Wilde EA, Li X2, Hunter JV et al. Loss of consciousness is related to white matter injury in mild traumatic brain injury. J. Neurotrauma 33 (22), 2000-2010 (2016).

70 Clark AL, Sorg SF, Schiehser DM et al. White matter associations with performance validity testing in veterans with mild traumatic brain injury: the utility of biomarkers in complicated assessment. J. Head Trauma Rehabil. 31(5), 346-59 (2016)

71 Jang SH, Yi JH, Kwon HG. Injury of the inferior cerebellar peduncle in patients with mild traumatic brain injury: a diffusion tensor tractography study. Brain Inj. 30(10), 1271-1275 (2016).

72 Mohammadian M, Roine T, Hirvonen J et al. High angular resolution diffusion-weighted imaging in mild traumatic brain injury. Neuroimage Clin. 17(13), 174-180 (2016).

73 Jarrett M, Tam R, Hernández-Torres E et al. A prospective pilot investigation of brain volume, white matter hyperintensities, and hemorrhagic lesions after mild traumatic brain injury. Front. Neurol. 7, 11 (2016).

74 Prabhu SP. The role of neuroimaging in sport-related concussion. Clin. Sports Med. 30, 103-114 (2011).

75 Wortzel HS, Kraus MF, Filley CM, Anderson CA, Arciniegas DB. Diffusion tensor imaging in mild traumatic brain injury litigation. J. Am. Acad. Psychiatry Law 39(4), 511-523 (2011).

76 Zappalà G, Thiebaut de Schotten M, Eslinger PJ. Traumatic brain injury and the frontal lobes: what can we gain with diffusion tensor imaging? Cortex 48, 156-165 (2012).

77 Aoki Y, Inokuchi R, Gunshin M, Yahagi N, Suwa H. Diffusion tensor imaging studies of mild traumatic brain injury: a meta-analysis. J. Neurol. Neurosurg. Psychiatry 83(9), 870-876 (2012).

78 Gardner A, Kay-Lambkin F, Stanwell P et al. A systematic review of diffusion tensor imaging findings in sports-related concussion. J. Neurotrauma 29(16), 2521-2538 (2012).

79 Bigler ED. Neuroimaging biomarkers in mild traumatic brain injury (mTBI). Neuropsychol. Rev. 23, 169-209 (2013).

80 Eierud C, Craddock RC, Fletcher $S$ et al. Neuroimaging after mild traumatic brain injury: review and meta-analysis. Neuroimage Clin. 4, 283-294 (2014).

81 Gardner A, Iverson GL, Stanwell P. A systematic review of proton magnetic resonance spectroscopy findings in sportrelated concussion. J. Neurotrauma 31(1), 1-18 (2014).

82 Keightley ML, Sinopoli KJ, Davis KD et al. Is there evidence for neurodegenerative change following traumatic brain injury in children and youth? A scoping review. Front. Hum. Neurosci. 8, 139 (2014).

83 Dimou S, Lagopoulos J. Toward objective markers of concussion in sport: a review of white matter and neurometabolic changes in the brain after sports-related concussion. J. Neurotrauma 31(5), 413-424 (2014).

84 Ashwal S, Tong KA, Ghosh N, Bartnik-Olson B, Holshouser BA. Application of advanced neuroimaging modalities in pediatric traumatic brain injury. J. Child Neurol. 29(12), 1704-1717 (2014).
85 Shin SS, Pathak S, Presson N et al. Detection of white matter injury in concussion using high-definition fiber tractography. Prog. Neurol. Surg. 28, 86-93 (2014).

86 Strauss S, Hulkower M, Gulko E et al. Current clinical applications and future potential of diffusion tensor imaging in traumatic brain injury. Top. Magn. Reson. Imaging 24(6), 353-362 (2015).

87 Hetherington H, Bandak A, Ling G, Bandak FA. Advances in imaging explosive blast mild traumatic brain injury. Handb. Clin. Neurol. 127, 309-318 (2015).

88 Bigler ED. Neuropathology of mild traumatic brain injury: correlation to neurocognitive and neurobehavioral findings. In: Brain Neurotrauma: Molecular, Neuropsychological, and Rehabilitation Aspects. Kobeissy FH (Ed.). CRC Press/Taylor \& Francis, FL, USA (2015).

89 Bramlett HM, Dietrich WD. Long-term consequences of traumatic brain injury: current status of potential mechanisms of injury and neurological outcomes. J. Neurotrauma 32(23), 1834-48 (2015).

90 Studerus-Germann AM, Thiran JP, Daducci A, Gautschi OP. Diagnostic approaches to predict persistent posttraumatic symptoms after mild traumatic brain injury - a literature review. Int. J. Neurosci. 126(4), 289-298 (2016).

91 Aoki Y, Inokuchi R. A voxel-based meta-analysis of diffusion tensor imaging in mild traumatic brain injury. Neurosci. Biobehav. Rev. 66, 119-126 (2016).

92 Delouche A, Attyé A, Heck O et al. Diffusion MRI: pitfalls, literature review and future directions of research in mild traumatic brain injury. Eur. J. Radiol. 85(1), 25-30 (2016).

93 Talavage TM, Nauman EA, Leverenz LJ. The role of medical imaging in the recharacterization of mild traumatic brain injury using youth sports as a laboratory. Front. Neurol. 19(6), Article 273 (2016).

94 Armstrong RC, Mierzwa AJ, Marion CM, Sullivan GM. White matter involvement after TBI: clues to axon and myelin repair capacity. Exp. Neurol. 275(3), 328-333 (2016).

95 Khong E, Odenwald N, Hashim E, Cusimano MD. Diffusion tensor imaging findings in post-concussion syndrome patients after mild traumatic brain injury: a systematic review. Front. Neurol. 7, 156 (2016).

96 Koerte IK, Hufschmidt J, Muehlmann Marc, Lin AP, Shenton ME. In: Translational Research in Traumatic Brain Injury. Laskowitz D, Grant G, (Eds). CRC Press/Taylor and Francis Group, FL, USA (2016).

97 Flygt J, Djupsjö A, Lenne F, Marklund N. Myelin loss and oligodendrocyte pathology in white matter tracts following traumatic brain injury in the rat. Eur. J. Neurosci. 38(1), 2153-2165 (2013).

98 Brody DL, Benetatos J, Bennett RE, Klemenhagen KC, Mac Donald CL. The pathophysiology of repetitive concussive traumatic brain injury in experimental models; new developments and open questions. Mol. Cell. Neurosci. 66(Pt B), 91-98 (2015).

99 Fehily B, Fitzgerald M. Repeated mild traumatic brain injury: potential mechanisms of damage. Cell Transplant. doi:10.3727/096368916X692807 (2016) (Epub ahead of print). 
100 Alhilali LM, Yaeger K, Collins M, Fakhran S. Detection of central white matter injury underlying vestibulopathy after mild traumatic brain injury. Radiology 272(1), 224-232 (2014).

101 FitzGerald DB, Crosson BA. Diffusion weighted imaging and neuropsychological correlates in adults with mild traumatic brain injury. Int. J. Psychophysiol. 82(1), 79-85 (2011).

102 Narayana PA, Herrera JJ, Bockhorst KH et al. Chronic cocaine administration causes extensive white matter damage in brain: diffusion tensor imaging and immunohistochemistry studies. Psychiatry Res. 221(3), $220-230$ (2014).

103 Fozouni N, Chopp M, Nejad-Davarani SP et al. Characterizing brain structures and remodeling after TBI based on information content, diffusion entropy. PLoS ONE 8(10), e76343 (2013).

104 Hope T, Westlye LT, BjØrnerud A. The effect of gradient sampling schemes on diffusion metrics derived from probabilistic analysis and tract-based spatial statistics. Magn. Reson. Imaging 30 (3), 402-412 (2012).

105 Jones D, Knösche T, Turner R. White matter integrity, fiber count, and other fallacies: the do's and don'ts of diffusion MRI. Neuroimage 73, 239-254 (2013).

106 Liu X, Yang Y, Sun J et al. Reproducibility of diffusion tensor imaging in normal subjects: an evaluation of different gradient sampling schemes and registration algorithm. Neuroradiology 56(6), 497-510 (2014).

107 Dodd AB, Epstein K, Ling JM, Mayer AR. Diffusion tensor imaging findings in semi-acute mild traumatic brain injury. J. Neurotrauma 31(14), 1235-1248 (2014).

108 Barrio-Arranz G, de Luis-García R, Tristán-Vega A, MartínFernández M, Aja-Fernández S. Impact of MR acquisition parameters on DTI scalar indexes: a tractography based approach. PLoS ONE 10(10), e0137905 (2015).

109 Knösche TR, Anwander A, Liptrot M, Dyrby TB. Validation of tractography: comparison with manganese tracing. Hum. Brain Mapp. 36(10), 4116-4134 (2015).

110 Farquharson S, Tournier JD, Calamante F et al. White matter fiber tractography: why we need to move beyond DTI J. Neurosurg. 118(6), 1367-1377 (2013).

111 Yeh FH, Verstynen TD, Wang Y, Fernández-Miranda JC, Tseng WYI. Deterministic diffusion fiber tracking improved by quantitative anisotropy. PLoS ONE 8(11), e80713 (2013).

112 Christidi F, Karavasilis E, Samiotis K, Bisdas S, Papanikolaou N. Fiber tracking: a qualitative and quantitative comparison between four different software tools on the reconstruction of major white matter tracts. Eur. J. Radiol. Open 3, 153-161 (2016).

113 Steven AJ, Zhuo J, Melhem ER. Diffusion kurtosis imaging: an emerging technique for evaluating the microstructural environment of the brain. AJR Am. J. Roentgenol. 202(1), W26-W33 (2014).

114 Fieremans E, Jensen JH, Helpern JA. White matter characterization with diffusional kurtosis imaging. Neuroimage 58(1), 177-188 (2011).
115 Marrale M, Collura G, Brai M et al. Physics, techniques and review of neuroradiological applications of diffusion kurtosis imaging (DKI). Clin. Neuroradiol. 26(4), 391-403 (2016).

116 Tefera GB, Zhou Y, Juneja V, Narayana PA. Evaluation of fiber tracking from subsampled q-space data in diffusion spectrum imaging. Magn. Reson. Imaging 31(6), 820-826 (2013).

117 Reichenbach JR, Schweser F, Serres B, Deistung A. Quantitative susceptibility mapping: concepts and applications. Clin. Neuroradiol. 25(Suppl. 2), 225-230 (2015).

118 Yarnykh VL. Fast macromolecular proton fraction mapping from a single off-resonance magnetization transfer measurement. Magn. Reson. Med. 68(1), 166-178 (2012).

119 Alonso-Ortiz E, Levesque IR, Pike GB. MRI-based myelin water imaging: a technical review. Magn. Reson. Med. 73(1), 70-81 (2015).

120 Chang EY, Du J, Chung CB. UTE imaging in the musculoskeletal system. J. Magn. Reson. Imaging 41(4), 870-883 (2015).

121 Vollmer T, Signorovitch J, Huynh L et al. The natural history of brain volume loss among patients with multiple sclerosis: a systematic literature review and meta-analysis. J. Neurol. Sci. 357(1-2), 8-18 (2015).

122 Pini L, Pievani M, Bocchetta $\mathrm{M}$ et al. Brain atrophy in Alzheimer's disease and aging. Ageing Res. Rev. 30, 25-48 (2016).

123 Matsuda H. MRI morphometry in Alzheimer's disease. Ageing Res. Rev. 30, 17-24 (2016).

124 Dong Z. Proton MRS and MRSI of the brain without water suppression. Prog. Nucl. Magn. Reson. Spectrosc. 86-87, 65-79 (2015).

125 Bertholdo D, Watcharakorn A, Castillo M. Brain proton magnetic resonance spectroscopy: introduction and overview. Neuroimaging Clin. N. Am. 23(3), 359-380 (2013).

126 McKay J, Tkáč I. Quantitative in vivo neurochemical profiling in humans: where are we now? Int. J. Epidemiol. 45(5), 1339-1350 (2016).

127 Koerte IK, Ertl-Wagner B, Reiser M, Zafonte R, Shenton ME. White matter integrity in the brains of professional soccer players without a symptomatic concussion. JAMA 308(18), 1859-1861 (2012).

128 Hulkower MB, Poliak DB, Rosenbaum SB, Zimmerman ME, Lipton ML. A decade of DTI in traumatic brain injury: 10 years and 100 articles later. AJNR Am. J. Neuroradiol. 34(11), 2064-2074 (2013).

129 Hoffer ME, Szczupak M, Balaban C. Clinical trials in mild traumatic brain injury. J. Neurosci. Methods 272, 77-81 (2016).

130 Grossman EJ, Ge Y, Jensen JH et al. Thalamus and cognitive impairment in mild traumatic brain injury: a diffusional kurtosis imaging study. J. Neurotrauma 29(13), 2318-2327 (2012).

131 Stokum JA, Sours C, Zhuo J, Kane R, Shanmuganathan K, Gullapalli RP. A longitudinal evaluation of diffusion kurtosis imaging in patients with mild traumatic brain injury. Brain Inj. 29(1), 47-57 (2015). 
132 Morey RA, Haswell CC, Selgrade ES et al. Effects of chronic mild traumatic brain injury on white matter integrity in Iraq and Afghanistan war veterans. Hum. Brain Mapp. 34(11), 2986-2999 (2013).

133 van den Heuvel MP, Sporns O. An anatomical substrate for integration among functional networks in human cortex. J. Neurosci. 33(36), 14489-500 (2013).

134 Slobounov SM, Gay M, Zhang K et al. Alteration of brain functional network at rest and in response to YMCA physical stress test in concussed athletes: RsFMRI study. Neuroimage 55(4), 1716-1727 (2011).

135 Vergara VM, Mayer AR, Damaraju E, Kiehl KA, Calhoun $\mathrm{V}$. Detection of mild traumatic brain injury by machine learning classification using resting state functional network connectivity and fractional anisotropy. J. Neurotrauma 34(5), 1045-1053 (2017).

136 Mitra J, Shen KK, Ghose S et al. Statistical machine learning to identify traumatic brain injury (TBI) from structural disconnections of white matter networks. Neuroimage 129, 247-259 (2016).

137 Lu L, Cao H, Wei X, Li Y, Li W. Iron deposition is positively related to cognitive impairment in patients with chronic mild traumatic brain injury: assessment with susceptibility weighted imaging. Biomed. Res. Int. 2015, 470676 (2015).

138 Hasiloglu ZI, Albayram S, Selcuk H et al. Cerebral microhemorrhages detected by susceptibility-weighted imaging in amateur boxers. AJNR Am. J. NeuroRadiol. 32(1), 99-102 (2011).

139 Yuh EL, Mukherjee P, Lingsma HF et al. Magnetic resonance imaging improves 3 -month outcome prediction in mild traumatic brain injury. Ann. Neurol. 73(2), 224-235 (2013).

140 Benson RR, Gattu R, Sewick B et al. Detection of hemorrhagic and axonal pathology in mild traumatic brain injury using advanced MRI: implications for neurorehabilitation. Neurorehabilitation 31(3), 261-279 (2012).

141 Toth A, Kovacs N, Tamas V et al. Microbleeds may expand acutely after traumatic brain injury. Neurosci. Lett. 617, 207-212 (2016).

142 Herrera JJ, Bockhorst K, Kondraganti S, Stertz L, Quevedo J, Narayana PA. Acute white matter tract damage after frontal mild traumatic brain injury. J. Neurotrauma 34(2), 291-299 (2016).

143 Wright DK, Trezise J, Kamnaksh A et al. Behavioral, blood, and magnetic resonance imaging biomarkers of experimental mild traumatic brain injury. Sci. Rep. 6, 28713 (2016).

144 Jurick SM, Bangen KJ, Evangelista ND, Sanderson-Cimino M, Delano-Wood L, Jak AJ. Advanced neuroimaging to quantify myelin in-vivo: application to mild TBI. Brain Inj. 30 (12), 1452-1457 (2016).

145 Zhou Y, Kierans A, Kenul D et al. Mild traumatic brain injury: longitudinal regional brain volume changes. Radiology 267(3), 880-890 (2013).

146 Lannsjö M, Raininko R, Bustamante M, von Seth C, Borg J. Brain pathology after mild traumatic brain injury: an exploratory study by repeated magnetic resonance examination. J. Rehabil. Med. 45(8), 721-728 (2013).
147 Ross DE, Ochs AL, Seabaugh JM et al. Progressive brain atrophy in patients with chronic neuropsychiatric symptoms after mild traumatic brain injury: a preliminary study. Brain Inj. 26(12), 1500-1509 (2012).

148 Clark AL, Sorg SF, Schiehser DM, Luc N et al. Deep white matter hyperintensities affect verbal memory independent of PTSD symptoms in veterans with mild traumatic brain injury. Brain Inj. 30 (7), 864-871 (2016).

149 Lin AP, Liao HJ, Merugumala SK, Prabhu SP, Meehan WP 3rd, Ross BD. Metabolic imaging of mild traumatic brain injury. Brain Imaging Behav. 6(2), 208-223 (2012).

150 Johnson B, Zhang K, Gay M et al. Metabolic alterations in corpus callosum may compromise brain functional connectivity in MTBI patients: an 1H-MRS study. Neurosci. Lett. 509, 5-8 (2012).

151 Grossman EJ, Kirov II, Gonen O et al. N-acetyl-aspartate levels correlate with intra-axonal compartment parameters from diffusion MRI. Neuroimage 118, 334-343 (2015).

152 Maugans TA, Farley C, Altaye M, Leach J, Cecil KM. Pediatric sports-related concussion produces cerebral blood flow alterations. Pediatrics 129(1), 28-37 (2012).

153 Johnson K, Zhang M, Gay S et al. Alteration of brain default network in subacute phase of injury in concussed individuals: resting-state fMRI study. NeuroImage 59(1), 511-518 (2012).

154 Mayer AR, Mannell MV, Ling J, Gasparovic C, Yeo RA. Functional connectivity in mild traumatic brain injury. Hum. Brain Mapp. 32(11), 1825-1835 (2011).

155 van de Looij Y, Mauconduit F, Beaumont $\mathrm{M}$ et al. Diffusion tensor imaging of diffuse axonal injury in a rat brain trauma model. NMR Biomed. 25(1), 93-103 (2012).

156 Bennett RE1, Mac Donald CL, Brody DL. Diffusion tensor imaging detects axonal injury in a mouse model of repetitive closed-skull traumatic brain injury. Neurosci. Lett. 513(2), 160-165 (2012).

157 Donovan V, Kim C, Anugerah AK et al. Repeated mild traumatic brain injury results in long-term white-matter disruption. J. Cereb. Blood Flow Metab. 34(4), 715-723 (2014).

158 Laitinen T, Sierra A, Bolkvadze T, Pitkänen A, Gröhn O. Diffusion tensor imaging detects chronic microstructural changes in white and gray matter after traumatic brain injury in rat. Front. Neurosci. 9, 128 (2015).

159 Tu TW, Williams RA, Lescher JD, Jikaria N, Turtzo LC, Frank JA. Radiological-pathological correlation of diffusion tensor and magnetization transfer imaging in a closed head traumatic brain injury model. Ann. Neurol. 79(6), 907-920 (2016).

160 Budde MD, Janes L, Gold E, Turtzo LC, Frank JA. The contribution of gliosis to diffusion tensor anisotropy and tractography following traumatic brain injury: validation in the rat using Fourier analysis of stained tissue sections. Brain 134(Pt 8), 2248-2260 (2011).

161 Robinson S, Berglass JB, Denson JL et al. Microstructural and microglial changes after repetitive mild traumatic brain injury in mice. J. Neurosci. Res. 95(4), 1025-1035 (2016).

162 Begonia MT, Prabhu R, Liao J et al. Quantitative analysis of brain microstructure following mild blunt and blast trauma. J. Biomech. 47(15), 3704-3711 (2014). 
163 Long JA, Watts LT, Li W et al. The effects of perturbed cerebral blood flow and cerebrovascular reactivity on structural MRI and behavioral readouts in mild traumatic brain injury. J. Cereb. Blood Flow Metab. 35(11), 1852-1861 (2015).

164 Hutchinson EB, Schwerin SC, Radomski KL, Irfanoglu MO, Juliano SL, Pierpaoli CM. Quantitative MRI and DTI abnormalities during the acute period following $\mathrm{CCI}$ in the ferret. Shock 46(Suppl. 31), 167-176 (2016).

165 Singh K, Trivedi R, Devi MM, Tripathi RP, Khushu S. Longitudinal changes in the DTI measures, anti-GFAP expression and levels of serum inflammatory cytokines following mild traumatic brain injury. Exp. Neurol. 275(3), 427-435 (2016).

166 Tu TW, Lescher JD, Williams RA, Jikaria N, Turtzo LC, Frank JA. Abnormal injury response in spontaneous mild ventriculomegaly wistar rat brains: a pathological correlation study of diffusion tensor and magnetization transfer imaging in mild traumatic brain injury. J. Neurotrauma 34(1), 248-256 (2016).
167 Li W, Long JA, Watts L, Shen Q, Liu Y, Jiang Z, Duong TQ. Spatiotemporal changes in diffusion, T2 and susceptibility of white matter following mild traumatic brain injury. $N M R$ Biomed. 29(7), 896-903 (2016).

168 Dewitt DS, Perez-Polo R, Hulsebosch CE, Dash PK, Robertson CS. Challenges in the development of rodent models of mild traumatic brain injury. J. Neurotrauma 30 (9), 688-701 (2013).

169 Levin HS, Robertson CS. Mild traumatic brain injury in translation. J. Neurotrauma 30 (8), 610-617 (2013).

170 Petraglia AL, Dashnaw ML, Turner RC, Bailes JE. Models of mild traumatic brain injury: translation of physiological and anatomic injury. Neurosurgery 75(Suppl. 4), S34-S49 (2014).

171 Shultz SR, McDonald SJ, Vonder Haar C et al. The potential for animal models to provide insight into mild traumatic brain injury: translational challenges and strategies. Neurosci. Biobehav. Rev. pii:S0149-7634 (2016) (Epub ahead of print).

172 Xiong Y, Mahmood A, Chopp M. Animal models of traumatic brain injury. Nat. Rev. Neurosci. 14(2), 128-142 (2013). 